\title{
Aircraft Noise Generation and Assessment
}

\author{
Installation - Numerical Investigation
}

\section{Lummer}

June 27th, 2016

\begin{abstract}
Acoustic installation effects are considered as scattering problem. Two methods to investigate them are presented. First, a Fast Multipole Method Boundary Element Method (FMM-BEM) which can solve the Helmholtz wave equation for full scale aircraft configurations at frequencies of some kHz. There, low Mach number potential mean flow fields can be taken into account by a so-called Taylor transformation. Second, a Discontinuous Galerkin Method (DGM) which solves Acoustic Perturbation Equations (APE) for realistic mean flow fields is presented. DGM calculations are very expensive and can be performed for full scale aircrafts only at low frequencies. Its main purpose so far is to assess the accuracy of the FMM for selected cases.

The Taylor transformed Helmholtz equation is derived and the fundamentals of the FMM are introduced. Some details of the DLR FMM code FMCAS are given. The basic DGM equations are derived for the APE and some implementation details of the DLR DGM code DISCO++ are discussed. For generic geometries results of the FMM and DGM are compared and the limits of the Taylor transfor-
\end{abstract}

M. Lummer

Department of Technical Acoustics,

Institute of Aerodynamics and Flow Technology,

German Aerospace Center (DLR),

Braunschweig, Germany,

E-mail: markus.lummer@dlr.de mation are shown. Finally, scattering results for a $1 \mathrm{kHz}$ Monopole at a full scale aircraft geometry will be presented.

Keywords airframe noise - acoustic shielding · acoustic scattering · Discontinuous Galerkin Method · Fast Multipole Method

\section{Nomenclature}

$c_{0}(\mathbf{x}) \quad$ Mean flow sound speed - assumed to be constant

$k \quad=\frac{\omega}{c_{0}}$ Wave number

$\mathrm{M} \quad=\frac{v_{\infty}}{c_{0}}$ Mean flow Mach number

$R_{d} \quad$ Radius of directivity circle

$\mathbf{v}_{0}(\mathbf{x}) \quad=\nabla \Phi_{0}$ Mean flow velocity

$\mathbf{v}_{\infty} \quad=\left(c_{0} M, 0,0\right)$ Free stream mean flow vector

$v_{\infty} \quad=c_{0} M$ Reference velocity

$\lambda \quad$ Wavelength

$\rho_{0}(\mathbf{x}) \quad$ Mean flow density - assumed to be constant

$\theta \quad$ Angular position on directivity circle

$\omega$ Circular frequency of acoustic disturbances

FMM specific symbols 


\begin{tabular}{|c|c|}
\hline $\mathscr{B}_{x}$ & $=1+\alpha \frac{\partial}{\partial n_{x}}$ Burton-Miller operator \\
\hline $\mathscr{C}_{x}$ & $=\mathscr{B}_{x} / 2$ on surface \\
\hline $\mathscr{D}_{y}$ & Shorthand for operator $\frac{\partial}{\partial n_{v}}-Y(\mathbf{y})$ \\
\hline$F_{\mathbf{a}}(\hat{\mathbf{s}})$ & FMM far-field signature function \\
\hline$G(\mathbf{x}, \mathbf{y})$ & Free field Green's function \\
\hline$h_{l}^{(1)}(z)$ & $\begin{array}{l}\text { Spherical Hankel function of the first } \\
\text { kind }\end{array}$ \\
\hline $\mathscr{K}_{\partial \Omega}(\mathbf{x})$ & $\begin{array}{l}\text { Integral operator (integration over sur- } \\
\text { face } \partial \Omega \text { ) }\end{array}$ \\
\hline$L$ & $\begin{array}{l}\text { Cut-off index of FMM transfer func- } \\
\text { tion }\end{array}$ \\
\hline$M_{L, \hat{\mathbf{s}}}(\mathbf{u})$ & FMM transfer function \\
\hline$N_{\mathbf{c}}(\hat{\mathbf{s}})$ & FMM near-field signature function \\
\hline $\mathbf{n}_{y}$ & Surface normal at point $\mathbf{y}$ \\
\hline $\mathscr{O}$ & Size of scattering surface $\partial \Omega$ \\
\hline$P_{l}(x)$ & Legendre Polynomials \\
\hline$p(\mathbf{x})$ & Fourier amplitude of acoustic pressure \\
\hline$\hat{\mathbf{s}}$ & Surface $\mathrm{p}$ \\
\hline $\mathscr{T}(\mathbf{x})$ & factor \\
\hline $\mathbf{v}(\mathbf{x})$ & $\begin{array}{l}=\nabla \phi \text { Fourier amplitude of acoustic } \\
\text { velocity }\end{array}$ \\
\hline$W_{j}^{m}$ & $\begin{array}{l}\text { Quadrature integration weight on sur- } \\
\text { face element } \Omega_{j}\end{array}$ \\
\hline $\mathbf{x}_{i}$ & Center point of surface element $\Omega_{i}$ \\
\hline$Y(\mathbf{x})$ & Wall admittance \\
\hline $\mathbf{y}_{j m}$ & $\begin{array}{l}\text { Quadrature collocation point on sur- } \\
\text { face element } \Omega_{j}\end{array}$ \\
\hline$\alpha$ & Burton-Miller constant \\
\hline$\Phi_{0}(\mathbf{x})$ & $\begin{array}{l}=v_{\infty} \Phi_{1}=c_{0} M \Phi_{1} \text { Mean flow velocity } \\
\text { potential }\end{array}$ \\
\hline$\Phi_{1}(\mathbf{x})$ & $\begin{array}{l}=\frac{\Phi_{0}}{V_{\infty}} \text { Mean flow velocity potential } \\
\text { scaled by reference velocity }\end{array}$ \\
\hline$\tilde{\phi}(\mathbf{x}, t)$ & Acoustic velocity potential \\
\hline$\phi(\mathbf{x})$ & $\begin{array}{l}=\mathscr{T} \psi \text { Fourier amplitude of acoustic } \\
\text { velocity potential }\end{array}$ \\
\hline$\psi(\mathbf{x})$ & $\begin{array}{l}=\mathscr{T}^{-1} \phi \text { Taylor-transformed Fourier } \\
\text { amplitude of acoustic velocity poten- } \\
\text { tial }\end{array}$ \\
\hline$\Omega$ & Computational domain \\
\hline$\partial\rfloor$ & Boundary of computational domain \\
\hline & Cube with center $\mathbf{c}$ \\
\hline
\end{tabular}

DGM specific symbols

$A_{\alpha \beta}^{j} \quad$ Flux matrices

$A_{\alpha \beta}^{j \mathfrak{n}} \quad$ Value of flux matrix at collocation point $\mathbf{x}_{\mathfrak{n}}$

$D_{\alpha \beta}^{p \mathfrak{n}} \quad=n_{j}^{p} A_{\alpha \beta}^{j \mathfrak{n}}=\mathbf{D}^{p \mathfrak{n}}$ Flux matrix on face

$\quad p$ and collocation point $\mathfrak{n}$

$F_{\alpha}^{j} \quad=A_{\alpha \beta}^{j} u_{\beta}$ Flux vectors

$F_{\alpha}^{p \mathfrak{n}} \quad=D_{\alpha \beta}^{p \mathfrak{n}} u_{\alpha}^{\mathfrak{n}}$ Flux vector on face $p$ and collocation point $\mathfrak{n}$

$H_{\alpha \beta}^{p \mathfrak{n} \pm} \quad=\mathbf{H}^{p \mathfrak{n} \pm}$ Upwind flux matrices

$\mathrm{M}(\mathbf{x}) \quad$ Galerkin test function

$\mathrm{N}_{\mathfrak{l}}(\mathbf{x}) \quad$ Lagrange interpolation polynomials

$\mathbf{R}^{p \mathfrak{n}} \quad$ Matrix of right eigenvectors of $\mathbf{D}^{p \mathfrak{n}}$ on face $p$ and collocation point $\mathfrak{n}$

$\mathbf{x}_{\mathfrak{n}} \quad$ Collocation points on tetrahedron

$\Lambda^{p \mathfrak{n}} \quad$ Matrix of eigenvalues on face $p$ and collocation point $\mathfrak{n}$

Super- and subscripts

$i, j, \ldots \quad$ Component of space vector

$\alpha, \beta, \ldots \quad$ Component of variable vector

$\mathfrak{l}, \mathfrak{m}, \ldots \quad$ Index of cell collocation point

\section{Contents}

1 Introduction ................ 2

2 From APE to the Helmholtz Equation . . . . 4

3 Solution of the Helmholtz Equation ... . . . . 5

4 Discontinuous Galerkin Method (DGM) . . . . . 10

5 Scattering by Generic Geometries . . . . . . . . . 12

6 Scattering by an Aircraft Geometry . . . . . . . . 16

7 Conclusions .............. 22

\section{Introduction}

For further reduction of community noise, engine installation effects should be taken into account already in the design phase of aircrafts. This requires sufficiently accurate numerical prediction tools, based preferably on the solution of the governing partial differential equations as provided by Computational Aeroacoustics (CAA). However, the solution of these equations is expensive because of the large difference of the involved length scales. While the acoustic wavelength is usually smaller than $1 \mathrm{~m}$, the length scale of the scattering 
geometry is in the order of some $10 \mathrm{~m}$, and the sound field must be evaluated at distances of several $100 \mathrm{~m}$. Even if a tight fitting Ffowcs WilliamsHawkings (FW-H) surface around the scattering geometry is used for the propagation of the sound into the far-field, CAA methods require very large volume meshes even for moderate frequencies of some kHz. Fortunately, for low Mach numbers, i. e., during the acoustically critical lift-off and approach phases, simplifications are possible that allow sufficiently accurate predictions of the acoustic installation effects. In this case, the governing equations can be reduced to a Helmholtz wave equation for a transformed acoustic velocity potential [47]. The Helmholtz equation can be solved very efficiently using special boundary element methods (BEM), so-called Fast Multipole Methods (FMM). The transformation was introduced by K. Taylor [47] assuming a low Mach number potential mean flow field. It has been used extensively in the investigation of shielding effects since then $[3,1,34,53$, 52].

Although CAA methods are very expensive, even now they can be used for validation purposes of lower fidelity methods at low to moderate frequencies (depending on the size of the scatterer). One of the most efficient CAA method concerning accuracy and geometrical flexibility is the Discontinuous Galerkin Method (DGM). Using a tetrahedral mesh, a quadrature free implementation is possible which performs well on current computer architectures.

In the present paper, emphasis is not layed on technical details of the algorithms, which can be found in the vast literature on the subject, but to make clear the physical and mathematical basis, as well as to present exemplary applications. Since the author is familiar with the DLR codes FMCAS (Fast Multipole Code for Acoustic Shielding) and DISCO++ (Discontinuous Galerkin Code in C++), the paper concentrates on their algorithms and implementation. It should, however, be mentioned that several other well validated and documented codes for acoustic shielding calculations are around Prominent examples, of which the author is aware of, are the Fast Scattering Code (FCS) developed at NASA Langley Research Center [48, 50,49, 18] and the ACTIPOLE code from Airbus Group Innovations $[17,8,9]$.

Two important topics which will not be discussed in the present paper are (1) sound absorbing boundary conditions, which are important for the assessment of passive noise control measures like, e. g., acoustic liners, and (2) the treatment of broadband noise problems with Boundary Element Methods.

Using the BEM/FMM it is quite straightforward to take into account sound absorbing boundary conditions by choosing an appropriate complex wall admittance, cf. eqn. (17). In [36], e. g., such values are determined by combination of calculations and experiments. The determination of sound absorbing boundary conditions for time domain calculations is more challenging. An overview of this topic can be found in $[39,40,45]$.

The calculation of broadband noise shielding problems using the classical FMM in frequency domain is an expensive task, since straightforward application would require a lot of calculations for the several frequency bands. A much more elegant approach is the development of time domain $\mathrm{BEM} / \mathrm{FMM}$ procedures as can be found in $[24,25$, 26].

This paper is structured as follows. Starting with an acoustic perturbation equation the Taylortransformed Helmholtz wave equation is derived. Then, the solution of the Helmholtz equation by a boundary integral equation (BIE) is given. The integral equation is solved by a boundary element method (BEM) which boils down to the solution of a large system of linear equations. For higher frequencies the linear system must be solved iteratively, requiring the evaluation of matrix-vector products. These matrix-vector products can be very efficiently performed using a Fast Multipole Method (FMM).

The second part of the paper deals with the DGM solution of Acoustic Perturbation Equations (APE) [19]. The APE are similar to the Linearized Euler Equations (LEE), but with non-acoustic parts of the solution removed. The DGM equations are derived for a tetrahedral mesh and unsteady boundary conditions as well as a newly developed hybrid 
approach, i. e., the coupling of unstructured meshes with structured cartesian blocks, are discussed.

Then, the FMM and DGM are applied to monopole scattering at a sphere and a plate, where the mean flow potential flow field is known. The limits of the Taylor transformation are shown. Finally, results for the scattering of a $1 \mathrm{kHz}$ monopole at a full scale aircraft geometry are presented, and the performance of FMM and DGM is compared. The paper closes with some conclusions.

\section{From APE to the Helmholtz Equation}

Sound waves are small disturbances of a mean flow field and thus linearized equations can be used to compute them. Usually, also viscous effects can be neglected and the most simple approach is to use Linearized Euler Equations (LEE). Unfortunately, also non-acoustic flow instabilities are solutions of the LEE and therefore specialized Acoustic Perturbation Equations (APE) [19,10] have been developed. The APE for the small pressure and velocity disturbances $\tilde{p}(\mathbf{x}, t)$ and $\tilde{\mathbf{v}}(\mathbf{x}, t)$ read

$$
\begin{aligned}
\frac{\partial \tilde{p}}{\partial t}+c_{0}^{2} \nabla \cdot\left(\rho_{0} \tilde{\mathbf{v}}+\mathbf{v}_{0} \frac{\tilde{p}}{c_{0}^{2}}\right) & =0, \\
\frac{\partial \tilde{\mathbf{v}}}{\partial t}+\nabla\left(\mathbf{v}_{0} \cdot \tilde{\mathbf{v}}+\frac{\tilde{p}}{\rho_{0}}\right) & =0 .
\end{aligned}
$$

$\rho_{0}(\mathbf{x})$ and $c_{0}(\mathbf{x})$ are the mean flow density and speed of sound and $\mathbf{v}_{0}(\mathbf{x})$ the mean flow velocity. In the following it is assumed that $\rho_{0}$ and $c_{0}$ are constant. The advantage of the APE, eqns. (1), is that the solution contains no vortical modes which can become unstable. The solution of the APE by the usual CAA methods is very expensive, but fortunately, for low Mach number potential mean flow fields, Taylor [47] found a method to reduce them to the classical Helmholtz equation for a transformed acoustic velocity potential. A very compact derivation of Taylor's transformation now follows.

Assuming a low Mach number potential mean flow field ${ }^{1} \mathbf{v}_{0}=\nabla \Phi_{0}$, introducing an acoustic velocity potential $\tilde{\mathbf{v}}=\nabla \tilde{\phi}$, as well as defining the convection operator $\mathscr{D}_{t} \equiv \frac{\partial}{\partial t}+\nabla \Phi_{0} \cdot \nabla$ reduces eqns.

\footnotetext{
1 The low Mach number assumption implies $\nabla \cdot \mathbf{v}_{0}=$ $\nabla^{2} \Phi_{0}=0$.
}

(1) to

$\mathscr{D}_{t} \tilde{p}+c_{0}^{2} \rho_{0} \nabla^{2} \tilde{\phi}=0, \quad \mathscr{D}_{t} \tilde{\phi}+\frac{\tilde{p}}{\rho_{0}}=0$.

Application of $\mathscr{D}_{t}$ to the second equation and substitution of the first one yields the following convected wave equation for the acoustic velocity potential

$\mathscr{D}_{t}^{2} \tilde{\phi}-c_{0}^{2} \nabla^{2} \tilde{\phi}=0$.

A low Mach number expansion of eqn. (3) can be made by introduction of a reference velocity $v_{\infty}$ and definition of a scaled mean flow potential $\Phi_{1} \equiv \frac{\Phi_{0}}{v_{\infty}}$ [47]. The Mach number then becomes $\mathbf{M} \equiv \frac{v_{\infty}}{c_{0}}$. Substituting $\Phi_{0}=c_{0} \mathrm{M} \Phi_{1}$ in eqn. (3) and dropping terms of $O\left(\mathrm{M}^{2}\right)$ yields the low Mach number convected wave equation

$\left(\frac{1}{c_{0}^{2}} \frac{\partial^{2}}{\partial t^{2}}+2 \frac{\mathrm{M}}{c_{0}} \nabla \Phi_{1} \cdot \nabla \frac{\partial}{\partial t}-\nabla^{2}\right) \tilde{\phi}=\tilde{S}$

where a source term $\tilde{S}(\mathbf{x}, t)$ has been added on the r.h.s. Now, the Taylor transformation [47] boils down to making the following ansatz for the acoustic potential

$$
\begin{array}{r}
\tilde{\phi}(\mathbf{x}, t)=e^{-i \omega t} e^{-i k \mathrm{M} \Phi_{1}(\mathbf{x})} \psi(\mathbf{x}), \\
\tilde{S}(\mathbf{x}, t)=S(\mathbf{x}) e^{-i \omega t} .
\end{array}
$$

$\psi(\mathbf{x})$ is the Taylor-transformed velocity potential, $k=\frac{\omega}{c_{0}}$ the wave number, and $i$ the imaginary unit. It is convenient to define a Taylor phase factor by $\mathscr{T}(\mathbf{x}) \equiv e^{-i k \mathrm{M} \Phi_{1}(\mathbf{x})}$. Substitution of eqn. (5) into the convected wave equation (4) yields

$\left(k^{2}+2 i k \mathrm{M} \nabla \Phi_{1} \cdot \nabla+\nabla^{2}\right) \psi \mathscr{T}=-S(\mathbf{x})$.

To proceed, one needs the derivatives of $\psi \mathscr{T}$

$$
\begin{aligned}
\nabla(\psi \mathscr{T}) & =\left(\nabla \psi-i k \mathrm{M} \psi \nabla \Phi_{1}\right) \mathscr{T}, \\
\nabla^{2}(\psi \mathscr{T}) & \approx\left(\nabla^{2} \psi-2 i k \mathrm{M} \nabla \psi \cdot \nabla \Phi_{1}\right) \mathscr{T} .
\end{aligned}
$$

In the second equation we have used $\nabla^{2} \Phi_{1}=0$ and dropped terms of $O\left(M^{2}\right)$. Substitution of eqns. (7) in eqn. (6) and dropping again terms $O\left(M^{2}\right)$ yields the Helmholtz equation for the function $\psi(\mathbf{x})$

$$
\left(k^{2}+\nabla^{2}\right) \psi=-S(\mathbf{x}) \mathscr{T}(\mathbf{x})^{-1} .
$$

An equation for the pressure can be obtained from the second equation of eqns. (2). The Fourier ansatz 
$\tilde{p}(\mathbf{x}, t)=p(\mathbf{x}) e^{-i \omega t}$ and $\tilde{\phi}(\mathbf{x}, t)=\phi(\mathbf{x}) e^{-i \omega t}$ yields the following equation for the pressure amplitude

$p(\mathbf{x})=\rho_{0}\left(i \omega-\nabla \Phi_{0} \cdot \nabla\right) \phi$.

At solid walls the normal velocity vanishes and the boundary condition of the velocity potential becomes $\frac{\partial \tilde{\phi}}{\partial n_{x}}=0$. Application of the operator $\frac{\partial}{\partial n_{x}}=$ $\mathbf{n}_{x} \cdot \nabla_{x}$ on eqn. (5) and inspection of eqn. (7) yields $\frac{\partial \psi}{\partial n_{x}}=0$ as boundary condition for the Taylor transformed potential at solid walls.

\section{Solution of the Helmholtz Equation}

The Helmholtz equation eqn. (8) is usually be solved by a boundary element method (BEM). There, using Kirchhoff's integral theorem [28], the Helmholtz equation is transformed into an integral equation. To obtain a unique solution a Burton-Miller approach is used. The integral equation is discretized on a surface triangulation and the solution of the resulting system of linear equations is accelerated by a Fast Multipole Method (FMM).

\subsection{The Kirchhoff Integral}

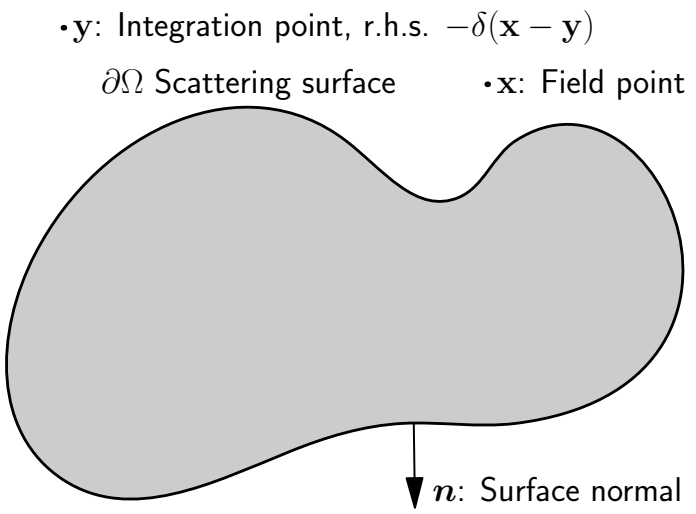

$\cdot \mathbf{z}$ : Source point, r.h.s $-\delta(\mathbf{y}-\mathbf{z})(-Q(\mathbf{z}))$

Fig. 1: Derivation of the Kirchhoff integral

The solution of the Helmholtz equation (8) can be given as so-called Kirchhoff integral over the boundary $\partial \Omega$ of the computational domain $\Omega$. We follow closely the derivation given in [41] and consider the Helmholtz equations for a field $\psi(\mathbf{y}, \mathbf{z})$ created by a point source of strength $Q(\mathbf{z})$ at a point $\mathbf{z}$ inside of the domain $\Omega$ (i. e. not on the boundary $\partial \Omega)$ and for the free field Green's function $G(\mathbf{x}, \mathbf{y})$, cf. Fig. 1,

$$
\begin{aligned}
& \left(\Delta+k^{2}\right) \psi(\mathbf{y}, \mathbf{z})=-\delta(\mathbf{y}-\mathbf{z})(-Q(\mathbf{z})), \\
& \left(\Delta+k^{2}\right) G(\mathbf{x}, \mathbf{y})=-\delta(\mathbf{x}-\mathbf{y}) .
\end{aligned}
$$

The solution of the second equation, i. e., the free field Green's function is

$G(\mathbf{x}, \mathbf{y})=\frac{e^{i k r}}{4 \pi r}, \quad r^{2}=(\mathbf{x}-\mathbf{y})^{2}$

Multiplication of the first equation of (10) with $G(\mathbf{x}, \mathbf{y})$ and of the second one with $\psi(\mathbf{y}, \mathbf{z})$, subtraction, and integration over the volume $\Omega(\mathbf{y})$ yields

$$
\begin{array}{r}
\int_{\Omega} \mathrm{d} \Omega_{y}[G(\mathbf{x}, \mathbf{y}) \Delta \psi(\mathbf{y}, \mathbf{z})-\psi(\mathbf{y}, \mathbf{z}) \Delta G(\mathbf{x}, \mathbf{y})]= \\
=\int_{\Omega} \mathrm{d} \Omega_{y} \delta(\mathbf{y}-\mathbf{z}) Q(\mathbf{z}) G(\mathbf{x}, \mathbf{y})+ \\
\quad+\int_{\Omega} \mathrm{d} \Omega_{y} \delta(\mathbf{x}-\mathbf{y}) \psi(\mathbf{y}, \mathbf{z}) .
\end{array}
$$

The evaluation of the $\delta$-functions on the r.h.s. yields

$$
\begin{array}{r}
\int_{\Omega} \mathrm{d} \Omega_{y}[G(\mathbf{x}, \mathbf{y}) \Delta \psi(\mathbf{y}, \mathbf{z})-\psi(\mathbf{y}, \mathbf{z}) \Delta G(\mathbf{x}, \mathbf{y})]= \\
=Q(\mathbf{z}) G(\mathbf{x}, \mathbf{z})+c \psi(\mathbf{x}, \mathbf{z}) .
\end{array}
$$

The constant $c$ is $1,1 / 2,0$ if the point $\mathbf{x}$ is inside of the volume, at the (smooth) boundary of the volume, or outside of the volume, respectively. Using Green's second identity, the volume integral on the left hand side of (13) can be reduced to an integral over the closed boundary of $\Omega$. We assume that one part of the boundary is at infinity, i. e. $|\mathbf{y}| \rightarrow \infty$, and denote the parts of the boundary in the finite region of $\Omega$ with $\partial \Omega$. Since the Green's function and its derivative vanish for $|\mathbf{y}| \rightarrow \infty$, Green's second identity yields the so-called Kirchhoff integral 
representation of $\psi(\mathbf{x}, \mathbf{y})$

$$
\begin{array}{r}
c \psi(\mathbf{x}, \mathbf{z})=(-Q(\mathbf{z})) G(\mathbf{x}, \mathbf{z})+ \\
+\int_{\partial \Omega} \mathrm{d} \Omega_{y}\left[\psi(\mathbf{y}, \mathbf{z}) \frac{\partial G(\mathbf{x}, \mathbf{y})}{\partial n_{y}}-G(\mathbf{x}, \mathbf{y}) \frac{\partial \psi(\mathbf{y}, \mathbf{z})}{\partial n_{y}}\right]
\end{array}
$$

where the surface normal points into the volume $\Omega$. Integration over the source volume $\mathbf{z}$ yields

$$
\begin{array}{r}
c \psi(\mathbf{x})=\psi_{V}(\mathbf{x})+ \\
+\int_{\partial \Omega} \mathrm{d} \Omega_{y}\left[\psi(\mathbf{y}) \frac{\partial G(\mathbf{x}, \mathbf{y})}{\partial n_{y}}-G(\mathbf{x}, \mathbf{y}) \frac{\partial \psi(\mathbf{y})}{\partial n_{y}}\right]
\end{array}
$$

where we have set for the volume integral

$$
\psi_{V}(\mathbf{x}) \equiv \int_{\Omega} \mathrm{d} \Omega_{z}(-Q(\mathbf{z})) G(\mathbf{x}, \mathbf{z})
$$

\subsection{The Boundary Integral Equation}

The acoustic field $\psi(\mathbf{x})$ which is produced by an incident field $\psi_{V}(\mathbf{x})$ scattered at the surface $\partial \Omega$ can be calculated from eqn. (15). In order to obtain an integral equation for the surface value $\psi(\mathbf{y})$, it is convenient to relate the gradient on the surface $\frac{\partial \psi(\mathbf{y})}{\partial n_{y}}$ to the value $\psi(\mathbf{y})$ by introduction of a given wall admittance $Y(\mathbf{y})$

$$
\frac{\partial \psi(\mathbf{y})}{\partial n_{y}}=Y(\mathbf{y}) \psi(\mathbf{y})
$$

At solid walls one has $Y=0$. Generally $Y$ is a complex number. Substitution of this relation into eqn. (15) and definition of the operator $\mathscr{D}_{y} \equiv \frac{\partial}{\partial n_{y}}-$ $Y(\mathbf{y})$ yields for a point $\mathbf{x}$ on the boundary $\partial \Omega$

$$
\begin{array}{r}
\frac{1}{2} \psi(\mathbf{x})-\int_{\partial \Omega} \mathrm{d} \Omega_{y} \psi(\mathbf{y}) \mathscr{D}_{y} G(\mathbf{x}, \mathbf{y})=\psi_{V}(\mathbf{x}), \\
\quad \mathbf{x} \in \partial \Omega .
\end{array}
$$

Unfortunately, the solution of this equation is not unique if the wave number $k$ is an eigenvalue of the inner problem of the body with surface $\partial \Omega$. A possible solution to this uniqueness problem is to consider a linear combination of eqn. (18) and a normal derivative of it with respect to $\mathbf{x}, \mathrm{cf}$. [13]. This so-called Burton-Miller approach is equivalent to the application of the operator $\mathscr{B}_{x} \equiv 1+\alpha \frac{\partial}{\partial n_{x}}$ to eqn. (18). $\alpha$ is the Burton-Miller coupling constant with $\mathfrak{I}(\alpha) \neq 0$. A common choice is $\alpha=\frac{i}{k}$. This is nearly optimal for sphere-like objects, cf. [2,29]. One obtains from eqn. (18)

$$
\begin{array}{r}
\frac{1}{2} \mathscr{B}_{x} \psi(\mathbf{x})-\mathscr{B}_{x} \int_{\partial \Omega} \mathrm{d} \Omega_{y} \psi(\mathbf{y}) \mathscr{D}_{y} G(\mathbf{x}, \mathbf{y})= \\
=\mathscr{B}_{x} \psi_{V}(\mathbf{x}) .
\end{array}
$$

It is convenient to introduce an integral operator $\mathscr{K}_{\partial \Omega}(\mathbf{x})$ for the integration over the surface $\partial \Omega$ by

$\mathscr{K}_{\partial \Omega}(\mathbf{x})(.) \equiv \int_{\partial \Omega} \mathrm{d} \Omega_{y}(.) \mathscr{D}_{y} G(\mathbf{x}, \mathbf{y})$.

Then, the boundary integral equation eqn. (19) can be written in the form

$\frac{1}{2} \mathscr{B}_{x} \psi(\mathbf{x})-\mathscr{B}_{x} \mathscr{K}_{\partial \Omega}(\mathbf{x}) \psi(\mathbf{y})=\mathscr{B}_{x} \psi_{V}(\mathbf{x})$.

The term $\mathscr{B}_{x} \psi(\mathbf{x})$ in the first term on the 1.h.s. of this equation contains a normal derivative $\frac{\partial \psi(\mathbf{x})}{\partial n_{x}}$ which can be expressed by $\psi(\mathbf{x})$ using eqn. (17)

$\mathscr{B}_{x} \psi(\mathbf{x})=\left[1+\alpha \frac{\partial}{\partial n_{x}}\right] \psi(\mathbf{x})=[1+\alpha Y(\mathbf{x})] \psi(\mathbf{x})$.

Defining $\mathscr{C}_{x} \equiv \frac{1}{2}[1+\alpha Y(\mathbf{x})]$ one obtains for the boundary integral equation

$\mathscr{C}_{x} \psi(\mathbf{x})-\mathscr{B}_{x} \mathscr{K}_{\partial \Omega}(\mathbf{x}) \psi(\mathbf{y})=\mathscr{B}_{x} \psi_{V}(\mathbf{x})$.

\subsubsection{Source Surfaces}

Aside from volume sources $\psi_{V}(\mathbf{x})$, it is also possible to consider so-called source surfaces, i. e., to prescribe values of $\psi$ and $\frac{\partial \psi}{\partial n}$ on some part $\partial \Omega_{s}$ of the integration boundary of eqn. (21). The integral equation (21) then becomes

$$
\begin{aligned}
& \mathscr{C}_{x} \psi(\mathbf{x})-\mathscr{B}_{x} \mathscr{K}_{\partial \Omega}(\mathbf{x}) \psi(\mathbf{y})= \\
= & \mathscr{B}_{x} \psi_{V}(\mathbf{x})+\mathscr{B}_{x} \mathscr{K}_{\partial \Omega_{s}}(\mathbf{x}) \psi_{s}(\mathbf{y}) .
\end{aligned}
$$

The Taylor-transformed potential $\psi_{s}(\mathbf{y})$ and its normal derivative on the source surface can be expressed by the acoustic pressure $p(\mathbf{y})$ and velocity $\mathbf{v}(\mathbf{y})$. Using $\phi=\mathscr{T} \psi$ the relation for $\psi_{s}$ can immediately be obtained from eqn. (9). In order to obtain the relation for $\nabla \psi_{s}$, one writes eqn. (9) in the form

$i k \phi=\frac{p}{\rho_{0} c_{0}}+\mathrm{M} \nabla \Phi_{1} \cdot \nabla \phi$ 
and substitutes it in the first equation of eqns. (7)

$$
\begin{aligned}
\nabla \phi=\nabla(\psi \mathscr{T}) & =\mathscr{T} \nabla \psi-i k \phi \mathrm{M} \nabla \Phi_{1} \approx \\
& \approx \mathscr{T} \nabla \psi-\frac{p}{\rho_{0} c_{0}} \mathrm{M} \nabla \Phi_{1}
\end{aligned}
$$

\subsection{Discretization}

For solution, the boundary integral equation (22) is usually be reduced to a system of linear equations for the surface values $\psi(\mathbf{x}), \mathbf{x} \in \partial \Omega$ by discretizing the surface $\partial \Omega=\bigcup_{j=0}^{N-1} \Omega_{j}$ in $N$ elements $\Omega_{j}$. Assuming a constant value $\psi_{j}$ on each element, the $\psi_{j}$ can be moved out of the integrals. Then, the $\mathscr{K}_{\Omega_{j}}(\mathbf{x})$ can be calculated by a quadrature formula with collocation points $\mathbf{y}_{j m}$ and weights $W_{j}^{m}$ on $\Omega_{j}$. One obtains

$$
\mathscr{K}_{\Omega_{j}}(\mathbf{x}) \approx \sum_{m} W_{j}^{m} \mathscr{D}_{y} G\left(\mathbf{x}, \mathbf{y}_{j m}\right)
$$

The resulting system of linear equations for the $\psi_{j}$ now reads

$$
\begin{array}{r}
\mathscr{C}_{x i} \psi_{i}-\mathscr{B}_{x i} \sum_{j=0}^{N-1} \psi_{j} \mathscr{K}_{\Omega_{j}}\left(\mathbf{x}_{i}\right)=\mathscr{B}_{x i} \psi_{V i}+ \\
+\mathscr{B}_{x i} \sum_{m=0}^{N_{s}-1} \psi_{s m} \mathscr{K}_{\Omega_{s m}}\left(\mathbf{x}_{i}\right), \quad i=0,1, \ldots, N-1 .
\end{array}
$$

It can be solved by direct or iterative methods. The point $\mathbf{x}_{i}$ can be chosen appropriately on each $\Omega_{i}$. Although iterative methods only require the calculation of matrix-vector products, eqn. (26) has a dense coefficient matrix which can't be calculated on the fly and must be stored just as for direct methods. Unfortunately, the number of unknowns $N$ required to solve a scattering problem for a surface of size $\mathscr{O}$ scales like $N \sim k^{2} \mathscr{O}$, where $k$ is the wave number of the acoustic field. Consequently, the required memory for the coefficient matrix is proportional to $k^{4}$ and becomes prohibitively large even for moderate wave numbers. Fast matrix-vector products avoiding the storage of the matrix are provided, e. g., by the Fast Multipole Method (FMM), cf. [15].

\subsection{Fast Multipole Method (FMM)}

The FMM for the Helmholtz equation comes in two flavours, a low- and a high-frequency version, depending on which kind of expansion is used for the free field Green's function [51]. In the lowfrequency regime the Green's function is approximated by an expansion into spherical harmonics (so-called partial wave expansion) and in the highfrequency regime the Green's function is written as an integral over the unit sphere (so-called plane wave expansion). Since it is easily possible to run a high-frequency FMM code as an ordinary BEM code at low frequencies, in the following we concentrate on the high-frequency FMM.

Bevor diving into technical details, the basic idea of the FMM is sketched. For simplicity, the linear system eqn. (26) is considered in the form $\sum_{j=0}^{N-1} B\left(\mathbf{x}_{i}, \mathbf{x}_{j}\right) \psi_{j}=b\left(\mathbf{x}_{i}\right), \quad i=0,1, \ldots, N-1$. To order the elements, the surface $\partial \Omega$ is covered by a set of cubes with diagonal $2 D$, cf. Fig. 2 . We denote the cube with center $\mathbf{c}$ by $\square_{\mathbf{c}}$ and wish to calculate the matrix-vector product for all equations with $\mathbf{x}_{i} \in \square_{\mathbf{c}} . \square_{\mathbf{c}}$ itself and the cubes adjacent to it, bluish in Fig. 2, form the near-field of $\square_{\mathbf{c}}$. The other cubes are its far-field. Now, we consider the partial sum of the matrix-vector product over all elements inside a cube $\square_{\mathbf{a}}$, i. e., $A(\mathbf{x})=\sum_{\mathbf{x}_{j} \in \square_{\mathbf{a}}} B\left(\mathbf{x}, \mathbf{x}_{j}\right) \psi_{j}$. For all cubes in the near field, $A\left(\mathbf{x}_{i}\right)$ is evaluated directly. The treatment of far-field cubes is essential for the FMM. For each far-field cube, the FMM approximates $A(\mathbf{x})$ by a so-called far-field signature function $F_{\mathbf{a}}(\hat{\mathbf{s}})$. Using a transfer function $M_{L, \hat{\mathbf{s}}}(\mathbf{c}-\mathbf{a})$, defined below, the $F_{\mathbf{a}}(\hat{\mathbf{s}})$ for all far-field cubes are then shifted and added up to a near-field signature function $N_{\mathbf{c}}(\hat{\mathbf{s}})=M_{L, \hat{\mathbf{s}}}(\mathbf{c}-\mathbf{a}) F_{\mathbf{a}}(\hat{\mathbf{s}})$ wich is defined in point $\mathbf{c}$. From this near-field signature function the partial sum $A\left(\mathbf{x}_{i}\right)$ from all far-field elements can be calculated.

In the following, the signatures and the transfer function will be constructed. Let the partial sum over the elements within a cube be denoted by $A(\mathbf{x})$ :

$$
\begin{array}{r}
A(\mathbf{x})=\sum_{\mathbf{x}_{j} \in \text { cube }} \psi_{j} \mathscr{K}_{\Omega_{j}}(\mathbf{x}) \approx \\
\approx \sum_{\mathbf{x}_{j} \in \text { cube }} \psi_{j} \sum_{m} W_{j}^{m} \mathscr{D}_{y} G\left(\mathbf{x}, \mathbf{y}_{j m}\right) .
\end{array}
$$




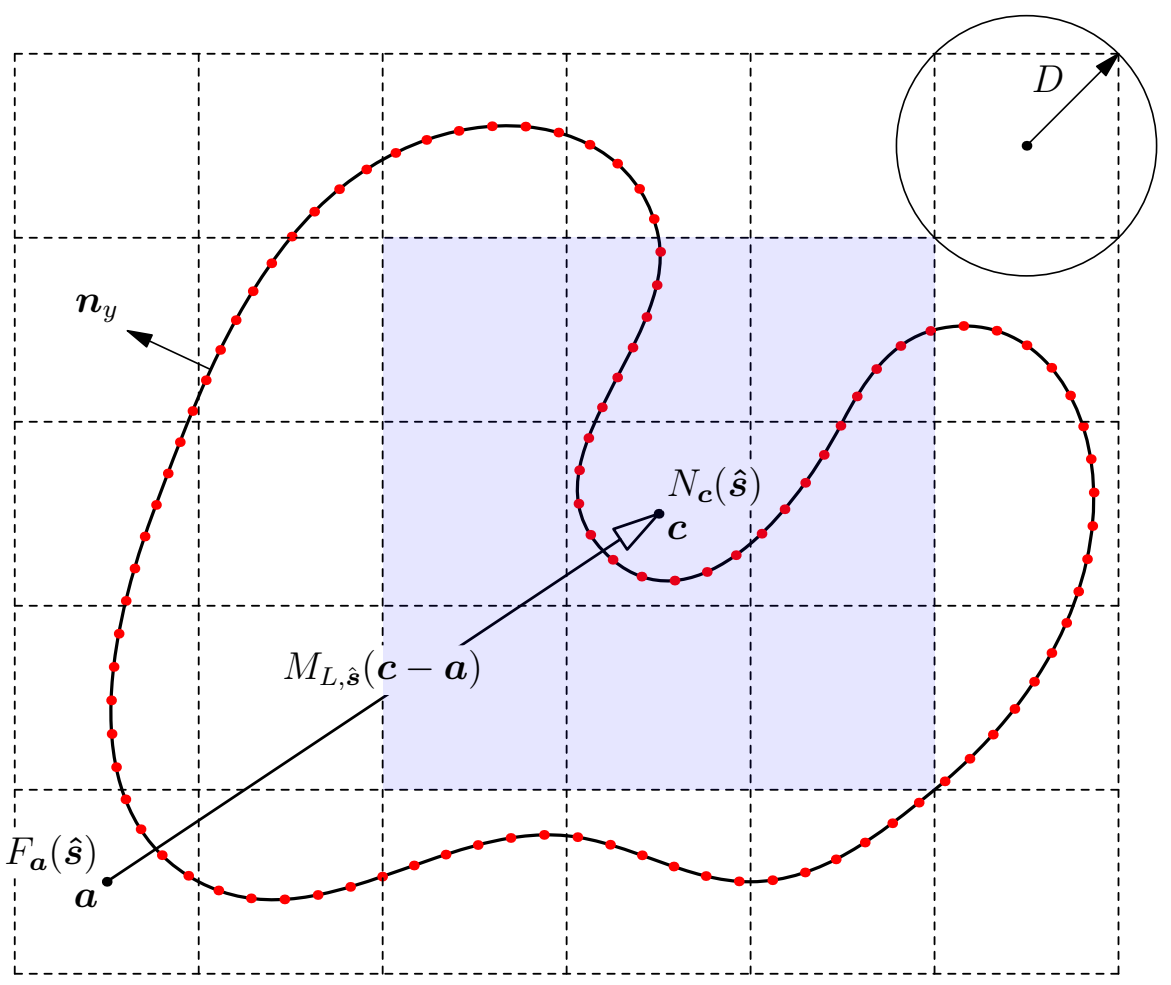

Fig. 2: Cube covering of geometry used for single level FMM. The diagonal of each cube is $2 D$. The red dots are surface collocation points. The bluish region is the geometrical near field of elements inside cube $\square$ c.

Now, an expansion is constructed, which can be evaluated very efficiently at points $\mathbf{x}$ with $\left|\mathbf{x}-\mathbf{y}_{j m}\right|>$ $D$, i. e., distant from the source region near the $\mathbf{y}_{j m}$. The following derivation refers heavily to the description given in [38]. First, the Green's function is approximated by a so-called plane wave expansion, given as integral over the unit sphere $S^{2}$

$$
\begin{aligned}
G(\mathbf{x}, \mathbf{y}) \approx & \frac{i k}{(4 \pi)^{2}} \int_{S^{2}} d \omega_{S} e^{i k \hat{\mathbf{s}} \cdot(\mathbf{x}-\mathbf{c})} \times \\
& \times M_{L, \hat{\mathbf{s}}}(\mathbf{c}-\mathbf{a}) e^{i k \hat{\mathbf{s}} \cdot(\mathbf{a}-\mathbf{y})} \\
|\mathbf{c}-\mathbf{a}| & >|(\mathbf{x}-\mathbf{c})+(\mathbf{a}-\mathbf{y})| .
\end{aligned}
$$

The unit vector $\hat{\mathbf{s}}$ is the integration variable pointing to the surface of the sphere and the points $\mathbf{a}$ and c are free parameters. $d \omega_{s}$ is the surface element of the sphere. Inspection of the integrand reveals, that any differential operator in $\mathbf{x}, \mathbf{y}$ applied to the Green's function acts only on the exponential func- tions under the integral and thus reduces to multiplication of the integrand by a complex number.

$M_{L, \hat{\mathbf{s}}}(\mathbf{u})$ is denoted as transfer function and has the form

$$
\begin{aligned}
& M_{L, \hat{\mathbf{s}}}(\mathbf{u})=\sum_{l=0}^{L}(2 l+1) i^{l} h_{l}^{(1)}(k u) P_{l}(\hat{\mathbf{s}} \cdot \hat{\mathbf{u}}), \\
& \mathbf{u}=u \hat{\mathbf{u}}, \quad|\hat{\mathbf{u}}|=1 .
\end{aligned}
$$

The $h_{l}^{(1)}(z)$ are spherical Hankel functions of the first kind and the $P_{l}(x)$ are Legendre polynomials. $L$ is a cut-off value which controls the accuracy of the approximation and must be properly determined, cf. $[15,14]$. The argument of the spherical Hankel function in eqn. (29) is proportional to the wave number $k$ and due to the singularity of $h_{l}^{(1)}(z)$ for $z \rightarrow 0$ the approximation breaks down for small wave numbers. Therefore, the plane wave approximation is not applicable in the low-frequency regime. 
Substitution of eqn. (28) into the partial sum, eqn. (27), yields

$$
\begin{aligned}
A_{\mathbf{a}}(\mathbf{x}) & \approx \frac{i k}{(4 \pi)^{2}} \int_{S^{2}} d \omega_{S} e^{i k \hat{\mathbf{s}} \cdot(\mathbf{x}-\mathbf{c})} \sum_{\mathbf{x}_{j} \in \mathrm{cube}} \psi_{j} \times \\
\times & \sum_{m} W_{j}^{m} D_{j m}(\hat{\mathbf{s}}) M_{L, \hat{\mathbf{s}}}(\mathbf{c}-\mathbf{a}) e^{i k \hat{\mathbf{s}} \cdot\left(\mathbf{a}-\mathbf{y}_{j m}\right)}
\end{aligned}
$$

Since the opereator $\mathscr{D}_{y}$ acts only on the exponential function it has been replaced by the factor $D_{j m}(\hat{\mathbf{s}}) \equiv$ $-\left[i k \mathbf{n}_{y} \cdot \hat{\mathbf{s}}+Y\left(\mathbf{y}_{j m}\right)\right]$. Now, the points a and $\mathbf{c}$ are clever specified. Setting $\mathbf{c}=\mathbf{x}$ gives

$$
\begin{aligned}
A_{\mathbf{a}}(\mathbf{x}) \approx \frac{i k}{(4 \pi)^{2}} \int_{S^{2}} d \omega_{S} & M_{L, \hat{\mathbf{s}}}(\mathbf{x}-\mathbf{a}) F_{\mathbf{a}}(\hat{\mathbf{s}}), \\
& |\mathbf{x}-\mathbf{a}|>\left|\mathbf{y}_{j m}-\mathbf{a}\right|, \\
F_{\mathbf{a}}(\hat{\mathbf{s}}) & \equiv \sum_{\mathbf{x}_{j} \in \operatorname{cube}} \psi_{j} \sum_{m} W_{j}^{m} \times \\
& \times D_{j m}(\hat{\mathbf{s}}) e^{i k \hat{\mathbf{s}} \cdot\left(\mathbf{a}-\mathbf{y}_{j m}\right)} .
\end{aligned}
$$

Since this approximation is valid for points outside of a region near point $\mathbf{a}$, the function $F_{\mathbf{a}}(\hat{\mathbf{s}})$ is called far-field signature. Setting $\mathbf{a}=\mathbf{y}_{j m}$ gives

$$
\begin{array}{r}
A_{\mathbf{a}}(\mathbf{x}) \approx \frac{i k}{(4 \pi)^{2}} \int_{S^{2}} d \omega_{S} e^{i k \hat{\mathbf{s}} \cdot(\mathbf{x}-\mathbf{c})} N_{\mathbf{c}}(\hat{\mathbf{s}}), \\
|\mathbf{x}-\mathbf{c}|<\left|\mathbf{y}_{j m}-\mathbf{c}\right|, \\
N_{\mathbf{c}}(\hat{\mathbf{s}}) \equiv \sum_{\mathbf{x}_{j} \in \text { cube }} \psi_{j} \sum_{m} W_{j}^{m} \times \\
\times D_{j m}(\hat{\mathbf{s}}) M_{L, \hat{\mathbf{s}}}\left(\mathbf{c}-\mathbf{y}_{j m}\right) .
\end{array}
$$

Since this approximation is valid in the vicinity of a point $\mathbf{c}$, the function $N_{\mathbf{c}}(\hat{\mathbf{s}})$ is called near-field signature. The definition of the near-field signature, eqn. (34), is usually not used in the FMM. Fundamental instead for the FMM is the following relation between the near-field and far-field signature functions. Substitution of eqn. (32) into eqn. (30) and comparison with eqn. (33) yields

$$
N_{\mathbf{c}}(\hat{\mathbf{s}})=M_{L, \hat{\mathbf{s}}}(\mathbf{c}-\mathbf{a}) F_{\mathbf{a}}(\hat{\mathbf{s}}) .
$$

Thus, using the transfer function the far-field signature near point $\mathbf{a}$ can be transformed into a nearfield signature near point $\mathbf{c}$. The partial sum $A_{\mathbf{a}}(\mathbf{x})$ near $\mathbf{c}$ can then be calculated by integration over the unit sphere using eqn. (33). The signature functions are represented by their values given at collocation points $\hat{\mathbf{s}}_{i j}$ on the unit sphere, where, e. g., $i$ represents an azimutal position and $j$ a polar position. The integral over the sphere must be performed with high accuracy. Usually one uses Gaussian collocations points in polar direction and equidistant points in azimuthal direction.

Signatures centered at different points can be related to each other. Setting $\mathbf{a}-\mathbf{y}_{j m}=(\mathbf{a}-\mathbf{b})+$ $\left(\mathbf{b}-\mathbf{y}_{j m}\right)$ in eqn. (32) gives the relation between the far-field signatures centered at $\mathbf{a}$ and $\mathbf{b}$

$F_{\mathbf{a}}(\hat{\mathbf{s}})=e^{i k \hat{\mathbf{s}} \cdot(\mathbf{a}-\mathbf{b})} F_{\mathbf{b}}(\hat{\mathbf{s}})$.

Setting $\mathbf{x}-\mathbf{c}=(\mathbf{x}-\mathbf{d})+(\mathbf{d}-\mathbf{c})$ in eqn. (33) gives the relation between the near-field signatures centered at $\mathbf{d}$ and $\mathbf{c}$

$N_{\mathbf{d}}(\hat{\mathbf{s}})=e^{i k \hat{\mathbf{s}} \cdot(\mathbf{d}-\mathbf{c})} N_{\mathbf{c}}(\hat{\mathbf{s}})$.

So far the basic ideas of the single level FMM have been presented, i. e., the FMM which uses transfer of the signature functions on one set of cubes having the same size. In doing so, the computational complexity of a matrix-vector product can be reduced from $O\left(N^{2}\right)$ to $O\left(N^{3 / 2}\right)$ [15].

\subsection{Multi Level FMM (MLFMM)}

Using a multi level FMM, the computational complexity of a matrix-vector product can further be reduced to $O(N \log N)$. In this case, the scattering geometry is put inside a cube, which is recursively subdivided into child cubes, $3^{3}=27$ sub cubes each, building a so-called octree. The far-field signatures of the child cubes $\square_{\mathbf{b}}$ can be moved up to the center of the parent cube $\square_{\mathbf{a}}$ by using eqn. (36) and the near-field signatures of a parent cube $\square_{\mathbf{c}}$ can be distributed to the centers of the child cubes $\square_{\text {d }}$ using eqn. (37). Moving the far-field signatures up the octree, their information content grows and more collocation points on the unit sphere are needed. Thus the signatures from the child cubes must be interpolated to the collocation points of the parent cube. Conversely, moving the near-field signatures down to the child cubes, they must be interpolated at the coarser distributed sphere points of the childs. An efficient and accurate interpolation method is a kind of spectral interpolation found, e. g., in [37]. 


\subsection{Implementation of the FMM}

A numerically efficient implementation of the MLFMM is quite complex and it is not possible to go into the details here. Since the DLR Code FMCAS is an almost standard implementation it is possible to refer to the literature, e. g., [44,54,53,33], and to mention only some points where special techniques have been found advantageous. First, the iterative solvers from the 'Portable, Extensible Toolkit for Scientific Computation' (PETSc) were used [6,5, 7]. In most cases the GMRES solver performed well. Second, the spectral interpolation method from [37] was used for interpolation between the different levels of the octree, cf. also [33]. Third, it was found that in dealing with very thin structures, a careful control of the numerical integration error in evaluation of the near-field surface integrals over the triangles is necessary. The reason for this is the singular nature of the integrands of the Burton-Miller equation, eqn. (20), which lead to accuracy problems if the triangles are ordered nearly back to back in case of thin structures, like, e. g., in case of wing spoilers. This error control could be achieved by using the DCUTRI software package [12], which provides an adaptive triangle integration. If the triangles are sufficiently far apart the triangle integration formulas from [30] can be used. Finally, the evaluation of the transfer function, eqn. (29), is very expensive and their function values must be stored. The transfer function depends on the two floating point numbers $k u$, and $\hat{\mathbf{s}} \cdot \hat{\mathbf{u}}$ from which a 64 bit key can be generated which allows simple storage of the function value in a std: : map C++ STL-container.

\section{Discontinuous Galerkin Method (DGM)}

The Discontinuous Galerkin Method (DGM) is a high order method for the solution of partial differential equations on unstructured meshes. The combination of high order approximation and geometrical flexibility makes it very well suited for CAA applications. In order to derive the DGM for the APE, it is convenient to use index notation and to introduce the acoustic variable vector $u_{\alpha}=(\tilde{p}, \tilde{\mathbf{v}})^{\top}, \alpha=0,1,2,3$ as well as to apply a summation convention over the latin space indices $i, j, \ldots$ and the greek variable indices $\alpha, \beta, \ldots$. The components of the mean flow velocity are denoted as $\mathbf{v}_{0}=\left(V_{0}, V_{1}, V_{2}\right)^{\top}$. The APE, eqn. (1), are written in the form

$$
\begin{aligned}
& \dot{u}_{\alpha}+F_{\alpha, j}^{j}-s_{\alpha}=0, \quad F_{\alpha}^{j}=A_{\alpha \beta}^{j} u_{\beta},
\end{aligned}
$$

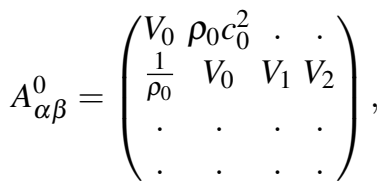

$$
\begin{aligned}
& A_{\alpha \beta}^{1}=\left(\begin{array}{cccc}
V_{1} & \cdot & \rho_{0} c_{0}^{2} & \cdot \\
\cdot & \cdot & \cdot & \cdot \\
\frac{1}{\rho_{0}} & V_{0} & V_{1} & V_{2} \\
\cdot & \cdot & . & .
\end{array}\right) \text {, } \\
& A_{\alpha \beta}^{2}=\left(\begin{array}{cccc}
V_{2} & \cdot & \cdot & \rho_{0} c_{0}^{2} \\
\cdot & \cdot & \cdot & \cdot \\
\cdot & \cdot & \cdot & \cdot \\
\frac{1}{\rho_{0}} & V_{0} & V_{1} & V_{2}
\end{array}\right) \text {, }
\end{aligned}
$$

where $F_{\alpha}^{j}$ are the flux vectors and $s_{\alpha}$ is a source term added. Multiplication of the APE with a test function $\mathrm{M}_{\mathfrak{m}}(\mathbf{x})$, integration over a volume $V$, and application of the Gaussian theorem yields the following weak formulation of the APE

$$
\begin{gathered}
\int_{V} \mathrm{~d} v \mathrm{M}_{\mathfrak{m}} \dot{u}_{\alpha}+\oint_{\partial V} \mathrm{~d} a n_{j} \mathrm{M}_{\mathfrak{m}} A_{\alpha \beta}^{j} u_{\beta}- \\
-\int_{V} \mathrm{~d} v \mathrm{M}_{\mathfrak{m}, j} A_{\alpha \beta}^{j} u_{\beta}-\int_{V} \mathrm{~d} v \mathrm{M}_{\mathfrak{m}} s_{\alpha}=0 .
\end{gathered}
$$

$n_{j}$ denotes the outer surface normal of the volume $V, d v$ is the volume element and $d a$ the surface element. A quadrature free implementation of the DGM can be obtained for volumes $V$ which can be mapped linearily to some unit cell [4]. This is the case for tetrahedral cells. The ansatz for a function, e. g., $u_{\alpha}(\mathbf{x}, t)$ on 'cubic' tetrahedral elements with 20 collocation points $\mathbf{x}_{\mathfrak{n}}, \mathfrak{n}=0,1, \ldots, 19$ is, cf. [55], $u_{\alpha}(\mathbf{x}, t)=\sum_{\mathfrak{n}=0}^{19} u_{\alpha}^{\mathfrak{n}}(t) \mathrm{N}_{\mathfrak{n}}(\mathbf{x})$, where the $u_{\alpha}^{\mathfrak{n}}(t)$ are the values of the variable vector at the $\mathbf{x}_{\mathfrak{n}}$ and the $\mathrm{N}_{\mathfrak{n}}(\mathbf{x})$ the corresponding Lagrange interpolation polynomials. Substitution of the Lagrange ansatz yields for the integrals over the volume of the tetra- 
hedron

$$
\begin{gathered}
\int_{V} \mathrm{~d} v \mathrm{M}_{\mathfrak{m}} \dot{u}_{\alpha}=\sum_{\mathfrak{n}=0}^{19} \dot{u}_{\alpha}^{\mathfrak{n}} \int_{V} \mathrm{~d} v \mathrm{M}_{\mathfrak{m}} \mathrm{N}_{\mathfrak{n}}, \\
\int_{V} \mathrm{~d} v \mathrm{M}_{\mathfrak{m}, j} A_{\alpha \beta}^{j} u_{\beta}=\sum_{\mathfrak{n}=0}^{19} A_{\alpha \beta}^{j \mathfrak{n}} u_{\beta}^{\mathfrak{n}} \int_{V} \mathrm{~d} v \mathrm{M}_{\mathfrak{m}, j} \mathrm{~N}_{\mathfrak{n}},
\end{gathered}
$$

where $A_{\alpha \beta}^{j \mathfrak{n}}$ is the value of the matrix $A_{\alpha \beta}^{j}$ at the point $\mathbf{x}_{\mathfrak{n}}$. For the integral over the surface of the tetrahedron one gets

$$
\begin{aligned}
& \oint_{\partial V} \mathrm{~d} a n_{j} \mathrm{M}_{\mathfrak{m}} A_{\alpha \beta}^{j} u_{\beta}=\sum_{p=0}^{3} \sum_{\mathfrak{n} \in\left\{\mathfrak{n}_{p}\right\}} n_{j}^{p} A_{\alpha \beta}^{j \mathfrak{n}} u_{\beta}^{\mathfrak{n}} \times \\
& \quad \times \int_{A_{p}} \mathrm{~d} a \mathrm{M}_{\mathfrak{m}} \mathrm{N}_{\mathfrak{n}},
\end{aligned}
$$

where $p$ is the index of the face and $n_{j}^{p}$ its normal. $\left\{\mathfrak{n}_{p}\right\}$ is the set of collocation points on face $p$. Now, we choose the test functions equal to the Lagrange polynomials, i. e. $M_{\mathfrak{m}}=N_{\mathfrak{m}}$, and define the $(20 \times 20)$ matrices

$$
\begin{gathered}
M_{\mathfrak{m} \mathfrak{n}}=\int_{V} \mathrm{~d} v \mathrm{~N}_{\mathfrak{m}} \mathrm{N}_{\mathfrak{n}}, \\
G_{\mathfrak{l n}}^{p}=\sum_{\mathfrak{m}=0}^{19} M_{\mathfrak{l m}}^{-1} \int_{A_{p}} \mathrm{~d} a \mathrm{~N}_{\mathfrak{m}} \mathrm{N}_{\mathfrak{n}}, \\
K_{\mathfrak{l n}}^{j}=\sum_{\mathfrak{m}=0}^{19} M_{\mathfrak{l m}}^{-1} \int_{V} \mathrm{~d} v \mathrm{~N}_{\mathfrak{m}, j} \mathrm{~N}_{\mathfrak{n}} .
\end{gathered}
$$

Multiplication of eqn. (40) from the left with $M_{\mathfrak{l m}}^{-1}$ yields the following system of ordinary differential equations for each tetrahedral cell

$$
\begin{array}{r}
\dot{u}_{\beta}^{\mathfrak{l}}+\sum_{p=0}^{3} \sum_{\mathfrak{n} \in\left\{\mathfrak{n}_{p}\right\}} G_{\mathfrak{l n}}^{p} \underbrace{n_{j}^{p} A_{\alpha \beta}^{j \mathfrak{n}} u_{\beta}^{\mathfrak{n}}}_{\equiv F_{\alpha}^{p \mathfrak{n}}}+ \\
+\sum_{\mathfrak{n}=0}^{19} K_{\mathfrak{l n}}^{j} A_{\alpha \beta}^{j \mathfrak{n}} u_{\beta}^{\mathfrak{n}}-s_{\alpha}^{\mathfrak{l}}=0 .
\end{array}
$$

The cells of the mesh are coupled by appropriately defined fluxes at the cell faces. Introducing the flux matrix $D_{\alpha \beta}^{j \mathfrak{n}} \equiv n_{j}^{p} A_{\alpha \beta}^{j \mathfrak{n}}$ one can write $F_{\alpha}^{p \mathfrak{n}}=D_{\alpha \beta}^{j \mathfrak{n}} u_{\beta}^{\mathfrak{n}}$ for the flux vector at face $p$. A common choice are Steger-Warming fluxes $[43,10]$ which read

$F_{\alpha}^{p \mathfrak{n}} \equiv D_{\alpha \beta}^{j \mathfrak{n}} u_{\beta}^{\mathfrak{n}}=H_{\alpha \beta}^{p \mathfrak{n}+} u_{\beta}^{\mathfrak{n}+}+H_{\alpha \beta}^{p \mathfrak{n}-} u_{\beta}^{\mathfrak{n}-}$.
Here, the face normal $n_{j}$ points out of the cell, the $u_{\beta}^{\mathfrak{n}+}$ denote the data values inside of the cell, and the $u_{\beta}^{\mathfrak{n}-}$ are the data values of the adjacent cell. The upwind matrices $H_{\alpha \beta}^{p \mathfrak{n} \pm}$ are calculated by a similarity transformation of the flux matrix $D_{\alpha \beta}^{p \mathfrak{n}} \equiv \mathbf{D}^{p \mathfrak{n}}$

$$
\mathbf{D}^{p \mathfrak{n}}=\mathbf{R}^{p \mathfrak{n}} \boldsymbol{\Lambda}^{p \mathfrak{n}}\left(\mathbf{R}^{p \mathfrak{n}}\right)^{-1},
$$

$\mathbf{H}^{p \mathfrak{n} \pm}=\frac{1}{2}\left[\mathbf{R}^{p \mathfrak{n}}\left(\boldsymbol{\Lambda}^{p \mathfrak{n}} \pm\left|\boldsymbol{\Lambda}^{p \mathfrak{n}}\right|\right)\left(\mathbf{R}^{p \mathfrak{n}}\right)^{-1}\right]$

where $\mathbf{R}^{p \mathfrak{n}}$ is the matrix of the right eigenvectors of $\mathbf{D}^{p \mathfrak{n}}, \boldsymbol{\Lambda}^{p \mathfrak{n}}$ is the diagonal matrix of the eigenvalues, and $\left|\boldsymbol{\Lambda}^{p \mathfrak{n}}\right|$ is the diagonal matrix of the modulus of of the eigenvalues. Using a simple rotation of the coordinate system the matrices $\mathbf{H}^{p \mathfrak{n} \pm}$ can be calculated analytically. Details can be found in [32].

The system of ordinary differential equations, eqn. (44), can be integrated by a standard 4th-order Runge-Kutta method.

\subsection{Boundary Conditions}

Standard boundary conditions of the DGM are solid wall conditions at impermeable walls and radiation conditions at outflow boundaries, cf. e.g., [10]. In order to provide arbitrary inflow boundary conditions, it is easily possible to attach ghost cells outside of boundary faces in which almost arbitrary unsteady pressure and velocity values can be prescribed. In this case, the flux through the face can be calculated like that for all other internal faces.

\subsection{Hybrid Meshes}

A major drawback of the DGM is its large memory requirement. For the presented implementation more than $6 \mathrm{~GB}$ memory are needed for $10^{6}$ tetrahedral cells representing the fields by single precision floating point numbers [32]. To circumvent this problem, it is possible to use hybrid meshes, $\mathrm{i}$. e., to combine unstructured tetrahedral meshes in the vicinity of the geometry with structured cartesian blocks in the far-field. The cartesian blocks are coupled with the unstructured mesh by covering groups of $3 \times 3 \times 3$ cartesian grid surface 
cells with 6 tetrahedra each. Then, from the outside, each cartesian block looks like an unstructured mesh and can easily be coupled to the fully unstructured mesh parts, provided that they have an appropriate regular triangulated surface mesh. Moreover, the outer cell layers provide a straightforward implementation of the radiation boundary condition. On the cartesian blocks the APE can be approximated with highly efficient 4th-order, 7-point Dispersion-Relation-Preserving (DRP) finite difference schemes [46]. Moreover, since the grid points of the cartesian blocks are collocation points of the tetrahedra, no interpolation between block and cell data is necessary. More details can be found in [32]. While the parallelization of purely unstructured meshes is easily done, using hybrid meshes a good load balancing between the several parallel processes is significantly harder to achieve. This must be investigated more thoroughly in future.

The generation of hybrid meshes must be automatized. In order to do so the computational domain is covered with a regular net of cubes. The size of each cube is the smallest cartesian block size. Next, the cubes containing parts of the geometry are marked. These cubes will be meshed unstructered. Then, the remaining cubes are combined into larger rectangular regions such that the interface between them becomes as small as possible. This can be done, e.g., by a suitable simulated annealing algorithm. Now, the surface meshes of the blocked region can be generated. Finally, the space between the surface of the blocked region and the scattering geometry is filled with tetrahedra. This can be done, e.g., by the open-source software Gmsh [23] or TetGen [42]. The set of hybrid blocks and the unstructured part of the mesh must then be distributed on the several MPI processes. Taking into account load balacing and minimum data exchange, this can be achieved using, e.g., the METIS software package [27]. So far, the implementation of the hybrid mesh generation in DISCO++ is quite experimental and a lot of room for optimization is expected.

\subsection{FW-H Surface}

DGM calculations are very expensive and the computational domain is usually chosen as small as possible. For the acoustical assessment of installation effects, however, the pressure field must be known far away from the aircraft. The standard technique to transfer the pressure information into the far-field is to use a so-called Ffowcs Williams-Hawkings (FW-H) surface which encloses the scattering geometry. Given pressure and velocity histories on the FW-H surface allows the reconstruction of the pressure signal by appropriate integration over the surface. This approach is based on the theory presented in [21]. Several different formulations are possible, cf., e.g., [20,22]. In DISCO++ a FW-H formulation from [35] is implemented, which takes into account explicitly a constant free stream velocity. The FW-H surface must be large enough to avoid regions with significant flow gradients.

\section{Scattering by Generic Geometries}

Now, the FMM and DGM are applied to generic geometries where the potential mean flow field is known and the limits of the Taylor transformation are examined. The first test case is the scattering of an acoustic monopole at a sphere with radius 1 located in the origin, and the second one, the scattering by a thin rectangular plate with dimensions $-1<x, y<1,|z|<0.02$. In the sphere case, the monopole is located on the $\mathrm{z}$-axis at $z=1.5$ and for the plate case, the monopole is located near a corner at $\mathbf{x}=(0.7,0.7,0.1)$. Both geometries are depicted in Fig. 3 where one also finds the definition of the directivity angle $\theta$. Although the generic test cases are quite small, the DGM calculations were performed using hybrid meshes. This is done to provide the reader with an impression of the hybrid mesh approach. To feed the monopole boundary conditions into the DGM calculation, the monopole positition was enclosed by a small spherical source surface. On this surface, pressure and velocity data from an acoustic monopole in constant mean flow [16] were applied. The monopole amplitude was scaled such that the free stream pressure in case of no mean flow was $p(r, t)=\exp (i k r-\omega t) / r$. Mean 


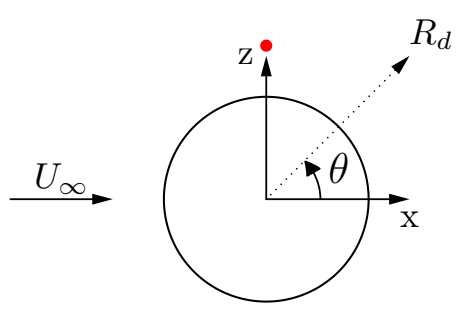

(a) Sphere

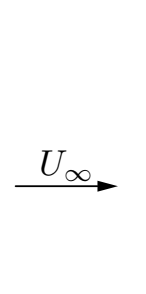

(b) Plate - (x,z) view

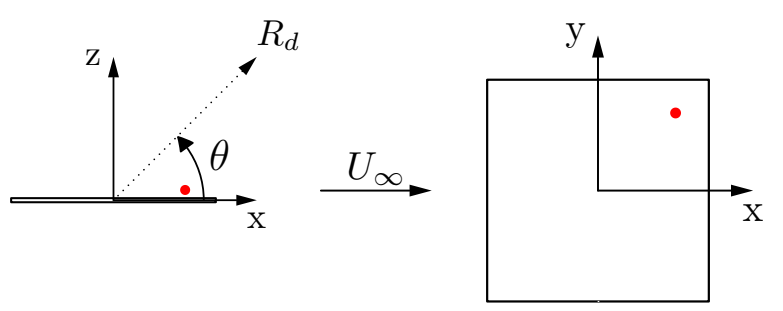

(c) Plate - (x,y) view

Fig. 3: Generic geometries for comparison of FMM and DGM. Monopole position marked red. $\theta$ is the parameter of the directivity circle (RMS value of pressure) with radius $R_{d}$.

flow density and sound speed were set to 1 in all cases.

In case of the sphere, the assumptions of a constant mean flow velocity at the source position is not exactly fulfilled, but seems to be justfied in view of the relatively small gradients there.

\subsection{Monopole Scattering by a Sphere}

In case of the unit sphere, DGM and FMM calculations for the scattering of an acoustic monopole with wavelength $\lambda=1.0,0.5$, and 0.25 and for mean flow Mach numbers $\mathrm{M}=0,0.1$, and 0.2 were performed. The mean flow potential for the flow around a unit sphere located in the origin is $(R=1$, $\left.r^{2}=\left(\mathbf{x}-\mathbf{x}_{0}\right)^{2}, \mathbf{x}_{0}=(0,0,0), \mathbf{v}_{\infty}=\left(c_{0} M, 0,0\right)\right)$

$\Phi_{0}(\mathbf{x})=\left(1+\frac{1}{2} \frac{R}{r^{3}}\right) \mathbf{v}_{\infty} \cdot\left(\mathbf{x}-\mathbf{x}_{0}\right)$.

The DGM source surface was a small sphere with radius 0.10 around the monopole position. Each wavelength was calculated with a different mesh, such that the resolution was about 6 points per wavelength (ppw) in case of DGM and about 9 elements per wavelength in case of FMM. The computational domain was the cube $-4<x, y, z<4$. The topology of the hybrid mesh in case of $\lambda=1.0$ is depicted in Fig. 4a and an (x,z)-cut through the mesh in Fig. 4b. The unstructured mesh around the sphere and the source surface around the monopole position is clearly visible. Near the source surface a higher mesh resolution was applied. Before the numerical details of the calculations are discussed, a survey of the results will be given. Fig.
5 depicts a $(\mathrm{x}, \mathrm{z})$ cut through the pressure field for $\lambda=0.25$ and the Mach numbers $\mathrm{M}=0,0.1,0.2$. Below of the sphere, along the negative $\mathrm{z}$-axis, the so-called Arago spot is visible. A small convective amplification in upstream direction is visible growing with increasing velocity. This becomes more evident looking at the directivity on a circle with radius $R_{d}=10$ in the (x,z) plane, cf. Fig. 6 to Fig. 8. In these plots the DGM and FMM results for the RMS value of the pressure are plotted together. For $\mathrm{M}=0$ and $\mathrm{M}=0.1$ both methods give nearly identical results. For $\mathrm{M}=0.2$ first small differences are visible. At larger Mach numbers, the Taylor-transformation quickly breaks down.

\subsubsection{Numerical Details}

All calculations were performed on the DLR CASE cluster $^{2}$. The parameters of the DGM calculations can be found inTable 1. The number of hybrid blocks were 19 for all calculations. The next two columns contain the number of tetrahedral cells for the coupling of the blocks and the radiation outflow conditions $\left(N_{B C}\right)$ as well as that of the unstructured core mesh $\left(N_{C}\right)$. Then, the degrees of freedom $(\mathrm{DOF})^{3}$ of the mesh is given. The size and number

2 The CASE cluster of the DLR Institute of Aerodynamics and Flow Technology in Braunschweig consists of 560 compute nodes with two Intel Xeon E5-2695V2 processors (IvyBridge architecture) and 128 GB RAM each. Each processor has a base frequency of $2.4 \mathrm{GHz}, 12$ cores, and 30 MB level 3 (L3) cache. Without oversubscription and Hyper-Threading 24 cores can be used on each node.

3 equivalent to the number of collocation points of the mesh. 


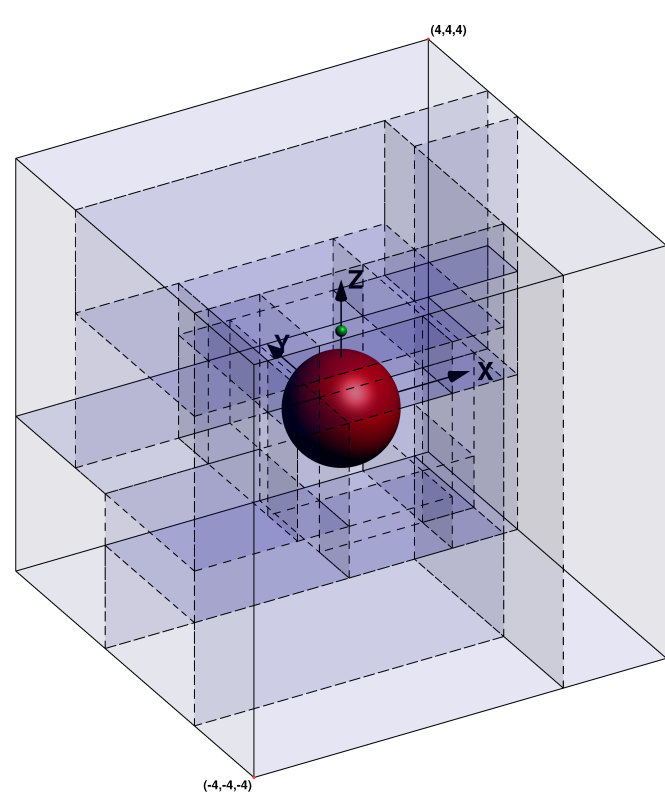

(a) Mesh Topology (19 blocks)

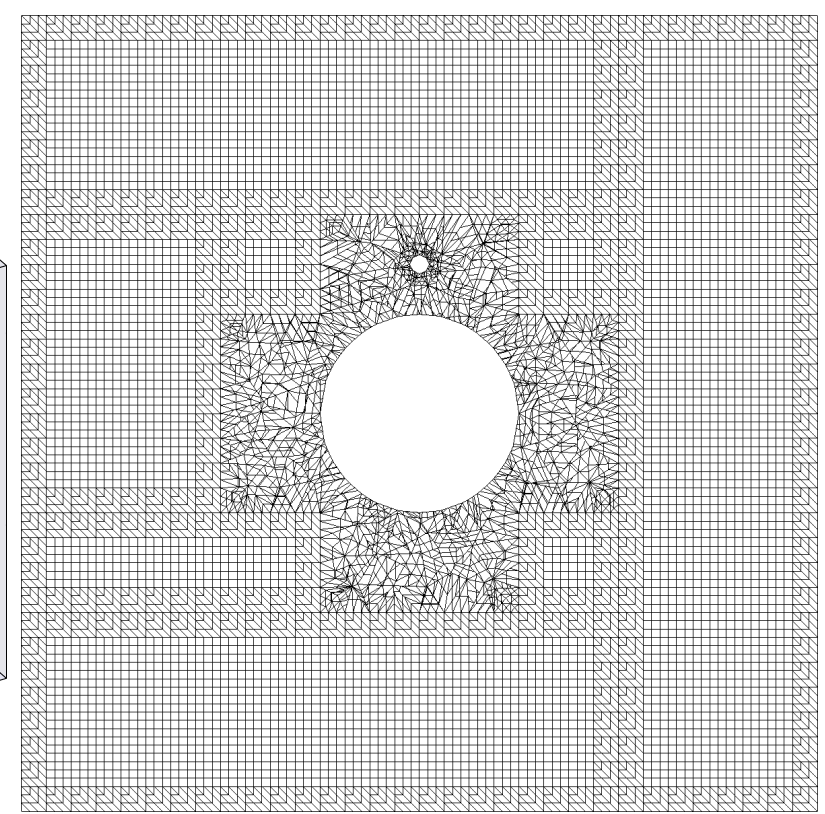

(b) (x,z)-cut through mesh

Fig. 4: Hybrid DGM mesh for sphere scattering (wavelength $\lambda=1.0$ )

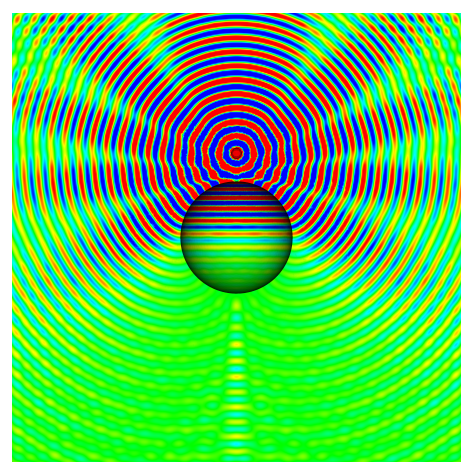

(a) $\mathrm{M}=0.0$

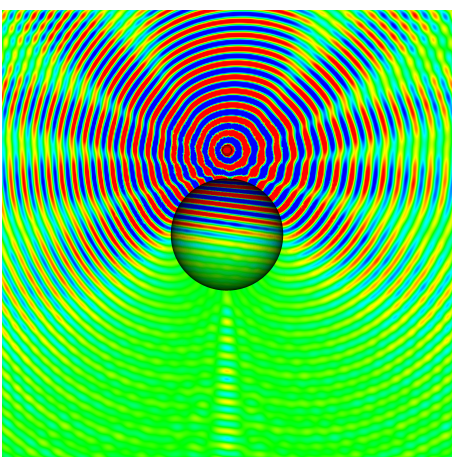

(b) $\mathrm{M}=0.1$

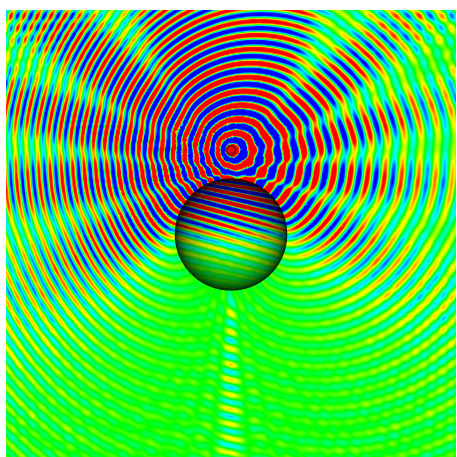

(c) $\mathrm{M}=0.2$

Fig. 5: DGM calculation for sphere scattering - $(\mathrm{x}, \mathrm{z})$-cut through pressure field, $\lambda=0.25$

Table 1: Parameters of DGM sphere calculations (4 Nodes)

\begin{tabular}{|l|c|r|r|r|r|r|r|r|r|}
\hline$\lambda$ & $N_{B}{ }^{\mathrm{a}}$ & $N_{B C}{ }^{\mathrm{b}}$ & $N_{C}{ }^{\mathrm{c}}$ & $D F^{\mathrm{d}}$ & $\Delta t$ & $N_{\text {Step }}$ & $\left.\mathrm{WCT}^{\mathrm{s}}\right]^{\mathrm{e}}$ & $\mathrm{M}[\mathrm{GB}]^{\mathrm{f}}$ & $\left.\mathrm{M}_{[\mathrm{GB}}\right]^{\mathrm{g}}$ \\
\hline 1.0 & 19 & 95044 & 8713 & 1113529 & $5.0 \mathrm{e}-03$ & 3200 & 559.6 & 1.76 & 2.75 \\
\hline 0.5 & 19 & 425092 & 54703 & 8092016 & $2.5 \mathrm{e}-03$ & 6400 & 4414.4 & 7.27 & 9.98 \\
\hline 0.25 & 19 & 1796356 & 399893 & 60244408 & $2.0 \mathrm{e}-03$ & 8000 & 24856.2 & 34.44 & 44.69 \\
\hline
\end{tabular}

${ }^{\mathrm{a}}$ Number of blocks. $\quad{ }^{\mathrm{b}}$ Number of block cells (coupling and radiation boundary).

${ }^{\mathrm{c}}$ Number of unstructured mesh cells. $\quad{ }^{\mathrm{d}}$ Degrees of freedom of whole mesh. $\quad{ }^{\mathrm{e}}$ Wall clock time.

${ }^{\mathrm{f}}$ Memory without FW-H storage. $\quad \mathrm{g}$ Memory with FW-H storage. 


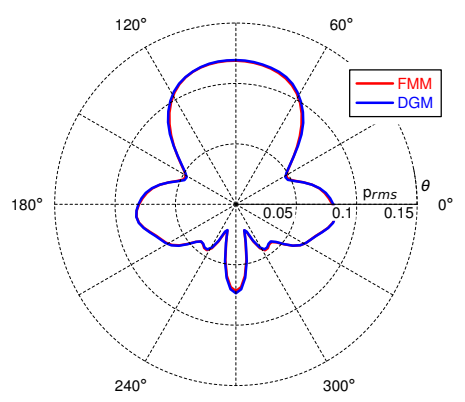

(a) $\mathrm{M}=0.0$

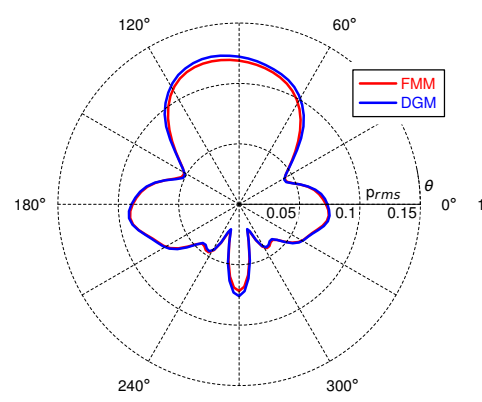

(b) $\mathrm{M}=0.1$

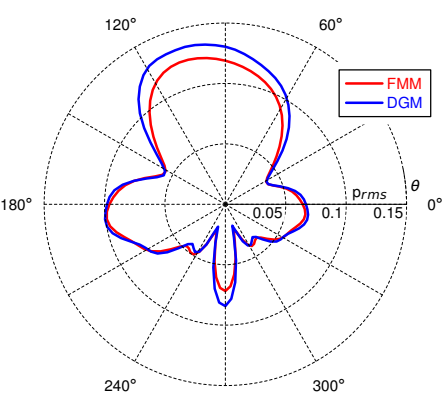

(c) $\mathrm{M}=0.2$

Fig. 6: Sphere directivity around y-axis $\lambda=1.0$

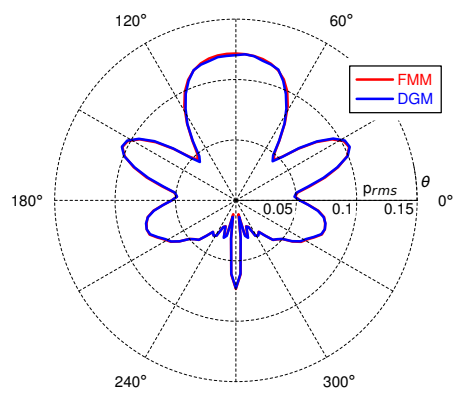

(a) $\mathrm{M}=0.0$

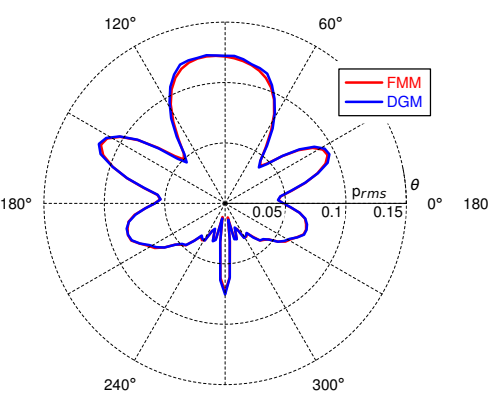

(b) $\mathrm{M}=0.1$

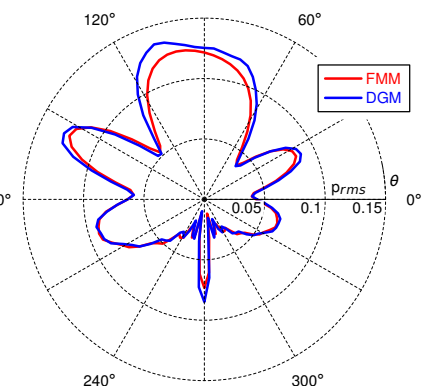

(c) $\mathrm{M}=0.2$

Fig. 7: Sphere directivity around y-axis $\lambda=0.5$

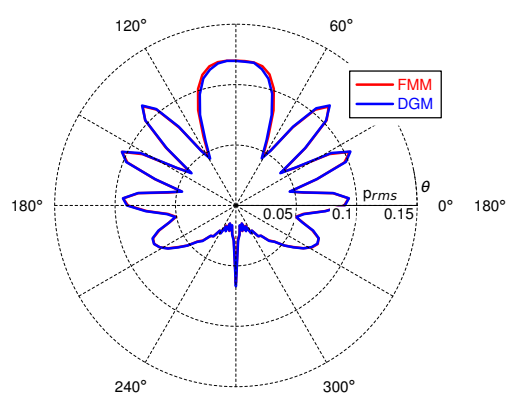

(a) $\mathrm{M}=0.0$

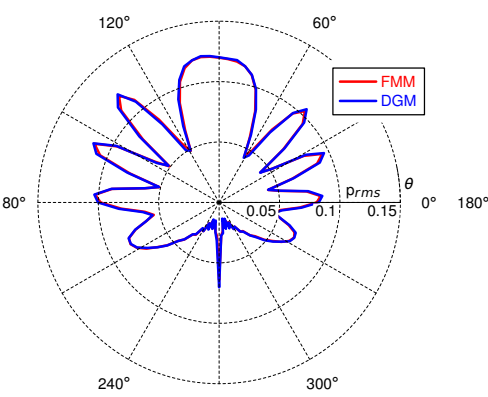

(b) $\mathrm{M}=0.1$

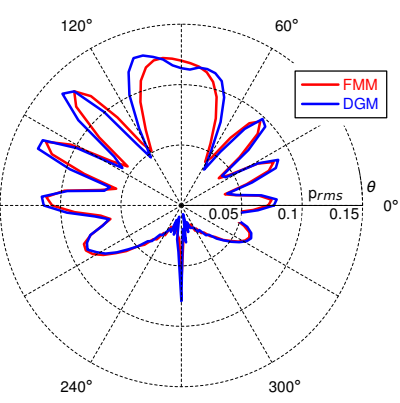

(c) $\mathrm{M}=0.2$

Fig. 8: Sphere directivity around y-axis $\lambda=0.25$

of the time steps as well as the Wall Clock Time (WCT) using 4 nodes (96 cores) follows. On each node 1 MPI process using 24 openMP threads was started. The last columns contain the memory used without and with storage of the time history on the FW-H surface. The FW-H surface was a sphere with radius 3. Characteristically for CAA methods the WCT and the memory requirements grow strongly nonlinear with decreasing wavelength. The shortest wave length calculation required about $7 \mathrm{~h}$ WCT and 45 GB memory. 
The parameters of the FMM calculations can be found inTable 2. First, the number of surface triangles for discretization of the scattering sphere is given. The resolution for all calculations is about 9 elements per wavelength and thus about $50 \%$ larger than required. The GMRES solver needed less than 10 iterations to converge. According to the experience of the author, such fast convergence is characteristic for almost convex geometries. Non convex geometries can require significant more iterations. Even for the shortest wave length the computational time was less than a minute and only about $250 \mathrm{MB}$ memory were required. Thus, the FMM is about a factor of 500 faster than the DGM.

\subsection{Monopole Scattering by a Plate}

In case of the thin plate, DGM and FMM calculations for the scattering of an acoustic monopole with wavelength $\lambda=1.0,0.5$, and 0.25 and for mean flow Mach numbers $\mathrm{M}=0,0.1$, and 0.2 were performed. Although the plate has a finite thickness the mean flow potential for a parallel flow was used $\Phi_{0}(\mathbf{x})=\mathbf{v}_{\infty} \mathbf{x}$. The DGM source surface was a small sphere with radius 0.04 around the monopole position. The monopole at position $(0.7,0.7,0.1)$ was placed asymmetric to the plate geometry and quite near to its upper surface. Each wavelength was calculated with a different mesh, such that the resolution was about 6 points per wavelength (ppw) in case of DGM and about 9 elements per wavelength in case of FMM. The computational domain was the cube $-3.5<x, y, z<3.5$. The topology of the hybrid mesh in case of $\lambda=1.0$ is depicted in Fig. 9a and an (x,z)-cut through the mesh in Fig. $9 \mathrm{~b}$. The unstructured mesh around the plate and the source surface can be identified. Around the source surface near the upper side of the plate a higher mesh resolution was used. Before the numerical details of the calculations are discussed a survey of the results will be given. Fig. 10 depicts a $(\mathrm{x}, \mathrm{z})$ cut through the pressure field for $\lambda=0.25$ and the Mach numbers $M=0,0.1,0.2$. With increasing velocity a small convective amplification in upstream direction is visible. This becomes more evident looking at the directivity of the pressure RMS data on a circle with radius $R_{d}=10$ in the $(\mathrm{x}, \mathrm{z})$ plane, cf. Fig. 11 to Fig. 13. In these plots the DGM and FMM results are plotted together. The fitting between DGM and FMM depends on the wavelength and is a little bit different from the sphere case. For $\lambda=0.5$ nearly a perfect match for all Mach numbers is achieved, cf. Fig. 12a to Fig. 12c, while for $\lambda=1.0$, cf. Fig. 11a to Fig. 11c, the DGM data are a little bit smaller and for $\lambda=0.25$, cf. Fig. 13a to Fig. 13c, a little bit larger than the FMM data. This suggests a small influence of the DGM mesh resolution. One should keep in mind that the DGM source surface is a mesh singularity located in close vicinity to a reflecting wall. This discrepancy deserves further investigations but nevertheless seems acceptable for technical purposes.

\subsubsection{Numerical Details}

The parameters of the DGM calculation can be found inTable 3. The number of hybrid blocks were 18 for all DGM calculations. Like in the sphere case 4 computational nodes with 24 cores were used. The overall performance is similar, but for the largest wave length $(\lambda=1.0)$ an unusual increase in WCT $(2314$ [s] for the plate vs. 560[s] for the sphere) can be observed. This could be a problem of a little bit clumsy distribution of the hybrid mesh on the MPI processes which was done fully automatic and is not optimized so far.

The parameters of the FMM calculation can be found inTable 4. Like expected, the performance of the FMM is more than one order of magnitude better than that of the DGM.

\section{Scattering by an Aircraft Geometry}

In order to demonstrate a large scale application, the scattering of an acoustic monopole at a medium size aircraft geometry is considered. Fig. 14 depicts the computational domain of the DGM. The length of the fuselage is about $32 \mathrm{~m}$ and the span about 29 $\mathrm{m}$. The wetted surface of the geometry is about 509 $\mathrm{m}^{2}$. The two engines are rear mounted at both sides of the fuselage. For the scattering calculations, the nacelles were removed and a monopole was placed at the right nacelle position. The wavelength of the calculations were about $\lambda=0.35 \mathrm{~m}$ corresponding 
Table 2: Parameters of FMM sphere calculations

\begin{tabular}{|l|r|r|r|r|r|}
\hline$\lambda$ & $N_{E}{ }^{\mathrm{a}}$ & $p p w^{\mathrm{b}}$ & $N_{\text {iter }}{ }^{\mathrm{c}}$ & $\left.\mathrm{WCT}^{\mathrm{S}}\right]^{\mathrm{d}}$ & $\mathrm{M}[\mathrm{MB}]^{\mathrm{e}}$ \\
\hline 1.00 & 1064 & 9.23 & 6 & 4.40 & 64.0 \\
\hline 0.50 & 4272 & 9.23 & 7 & 12.60 & 85.0 \\
\hline 0.25 & 16680 & 9.11 & 8 & 50.70 & 250.0 \\
\hline
\end{tabular}

${ }^{\mathrm{a}}$ Number of elements. ${ }^{\mathrm{b}}$ Elements per wavelength.

${ }^{\mathrm{c}}$ Number of GMRES iterations. $\quad{ }^{\mathrm{d}}$ Wall clock time on 1 node. ${ }^{\mathrm{e}}$ Memory.

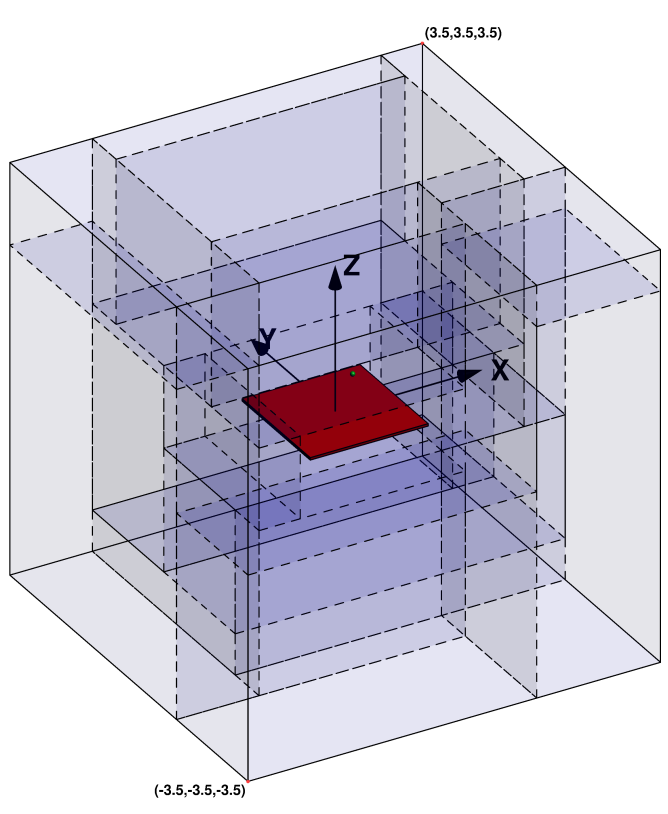

(a) Mesh Topology (18 blocks)

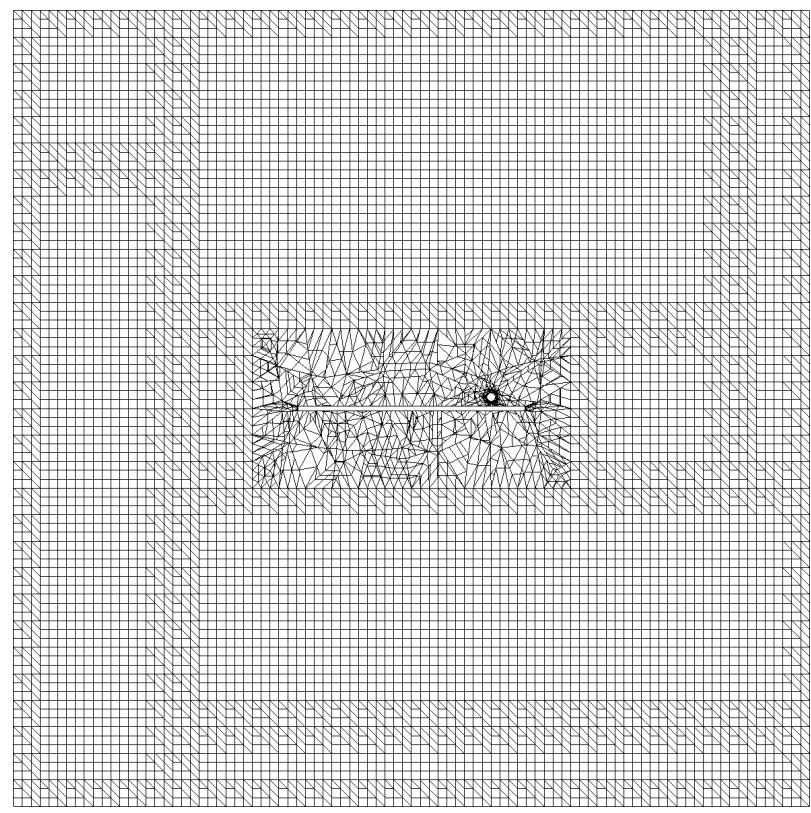

(b) $(\mathrm{x}, \mathrm{z})$-cut through mesh and source sphere

Fig. 9: Hybrid DGM mesh for plate scattering (wavelength $\lambda=1.0$ )

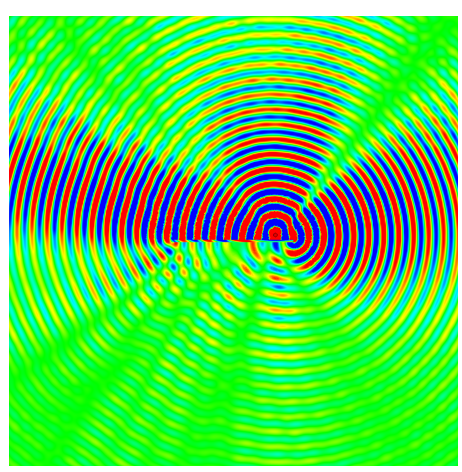

(a) $\mathrm{M}=0.0$

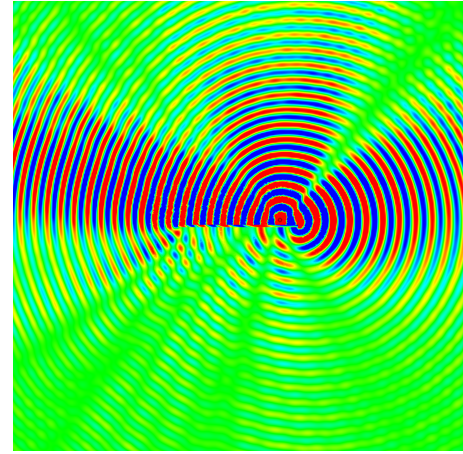

(b) $\mathrm{M}=0.1$

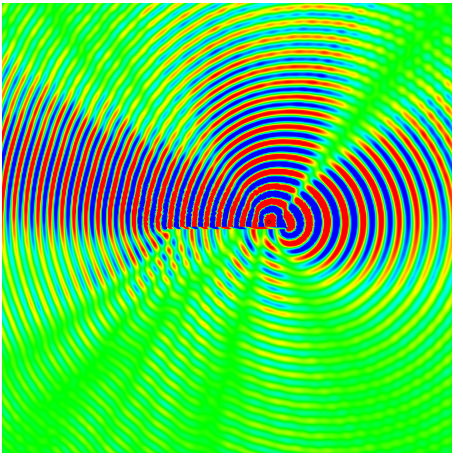

(c) $\mathrm{M}=0.2$

Fig. 10: DGM calculation for plate scattering $-(\mathrm{x}, \mathrm{z})$-cut through pressure field, $\lambda=0.25$ 


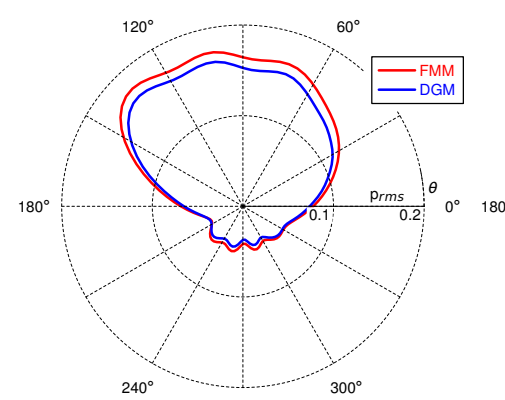

(a) $\mathrm{M}=0.0$

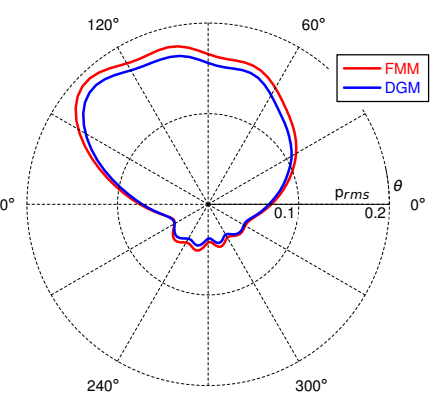

(b) $\mathrm{M}=0.1$

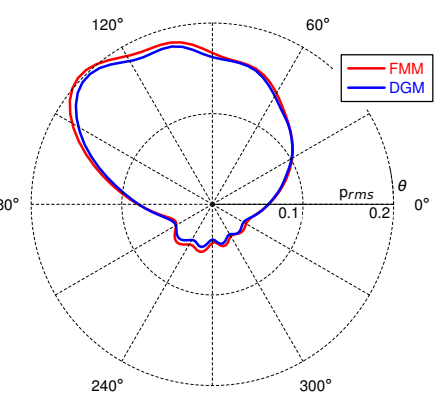

(c) $\mathrm{M}=0.2$

Fig. 11: Plate directivity around y-axis $\lambda=1.0$

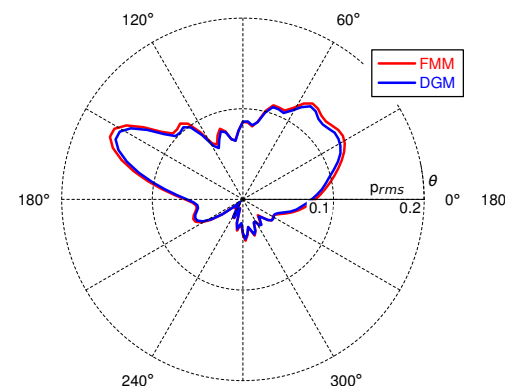

(a) $\mathrm{M}=0.0$

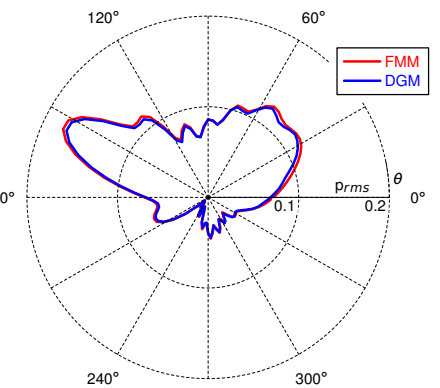

(b) $\mathrm{M}=0.1$

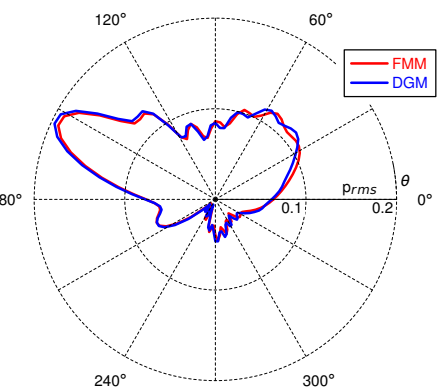

(c) $\mathrm{M}=0.2$

Fig. 12: Plate directivity around $y$-axis $\lambda=0.5$

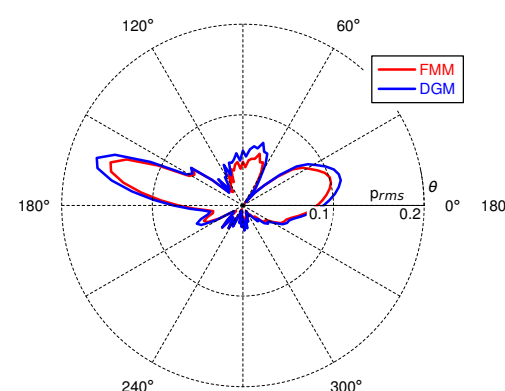

(a) $\mathrm{M}=0.0$

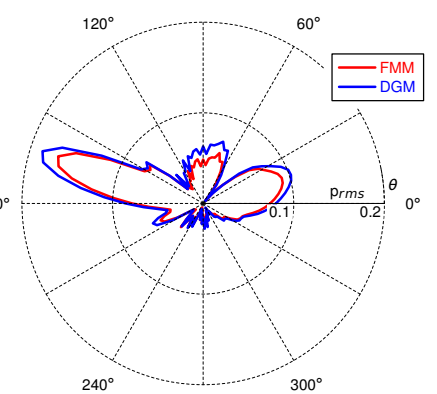

(b) $\mathrm{M}=0.1$

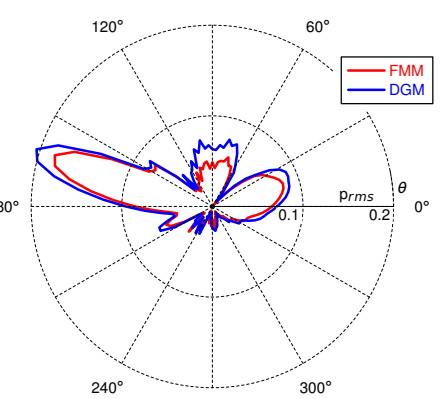

(c) $\mathrm{M}=0.2$

Fig. 13: Plate directivity around $y$-axis $\lambda=0.25$

to a frequency of about $1 \mathrm{kHz}$. No mean flow data were available and thus mean flow effects were not taken into a account.

\subsection{The DGM Calculation}

For the DGM calculation a hybrid mesh was used. The computational domain was the box $-10<x<$ $41,-21<y<21$, and $-10<z<11$. The nose of the aircraft is located in the origin, pointing in 
Table 3: Parameters of DGM plate calculations (4 Nodes)

\begin{tabular}{|c|c|c|c|c|c|c|c|c|c|}
\hline$\lambda$ & $N_{B}{ }^{\mathrm{a}}$ & $N_{B C}{ }^{\mathrm{b}}$ & $N_{C}{ }^{\mathrm{c}}$ & $D O F^{\mathrm{d}}$ & $\Delta t$ & $N_{\text {Step }}$ & $\mathrm{WCT}_{[\mathrm{s}]^{\mathrm{e}}}$ & $\mathrm{M}[\mathrm{GB}]^{\mathrm{f}}$ & $\mathrm{M}[\mathrm{GB}]^{\mathrm{g}}$ \\
\hline 1.0 & 18 & 94230 & 4596 & 952126 & $1.0 \mathrm{e}-03$ & 16000 & 2313.8 & 1.67 & 2.34 \\
\hline 0.5 & 18 & 425268 & 27043 & 6954196 & $1.5 \mathrm{e}-03$ & 10667 & 6950.8 & 6.74 & 9.45 \\
\hline 0.25 & 18 & 1803600 & 194091 & 51339843 & $2.0 \mathrm{e}-03$ & 8000 & 22978.2 & 31.09 & 41.09 \\
\hline
\end{tabular}

a Number of blocks. $\quad$ b Number of block cells (coupling and radiation boundary).

${ }^{\mathrm{c}}$ Number of unstructured mesh cells. $\quad{ }^{\mathrm{d}}$ Degrees of freedom of whole mesh. $\quad{ }^{\mathrm{e}}$ Wall clock time.

${ }^{\mathrm{f}}$ Memory without FW-H storage. $\quad$ g Memory with FW-H storage.

Table 4: Parameters of FMM plate calculations

\begin{tabular}{|l|r|r|r|r|r|}
\hline$\lambda$ & $N_{E}{ }^{\mathrm{a}}$ & \multicolumn{1}{c}{$p p w^{\mathrm{b}}$} & $N_{\text {iter }}{ }^{\mathrm{c}}$ & WCT [s] $^{\mathrm{d}}$ & $\mathrm{M}[\mathrm{MB}]^{\mathrm{e}}$ \\
\hline 1.00 & 848 & 10.10 & 7 & 30.30 & 60.0 \\
\hline 0.50 & 3060 & 9.59 & 9 & 113.90 & 89.0 \\
\hline 0.25 & 11408 & 9.26 & 11 & 425.10 & 227.0 \\
\hline
\end{tabular}

${ }^{\mathrm{a}}$ Number of elements. ${ }^{\mathrm{b}}$ Elements per wavelength.

${ }^{\mathrm{c}}$ Number of GMRES iterations. ${ }^{\mathrm{d}}$ Wall clock time on 1 node. ${ }^{\mathrm{e}}$ Memory.

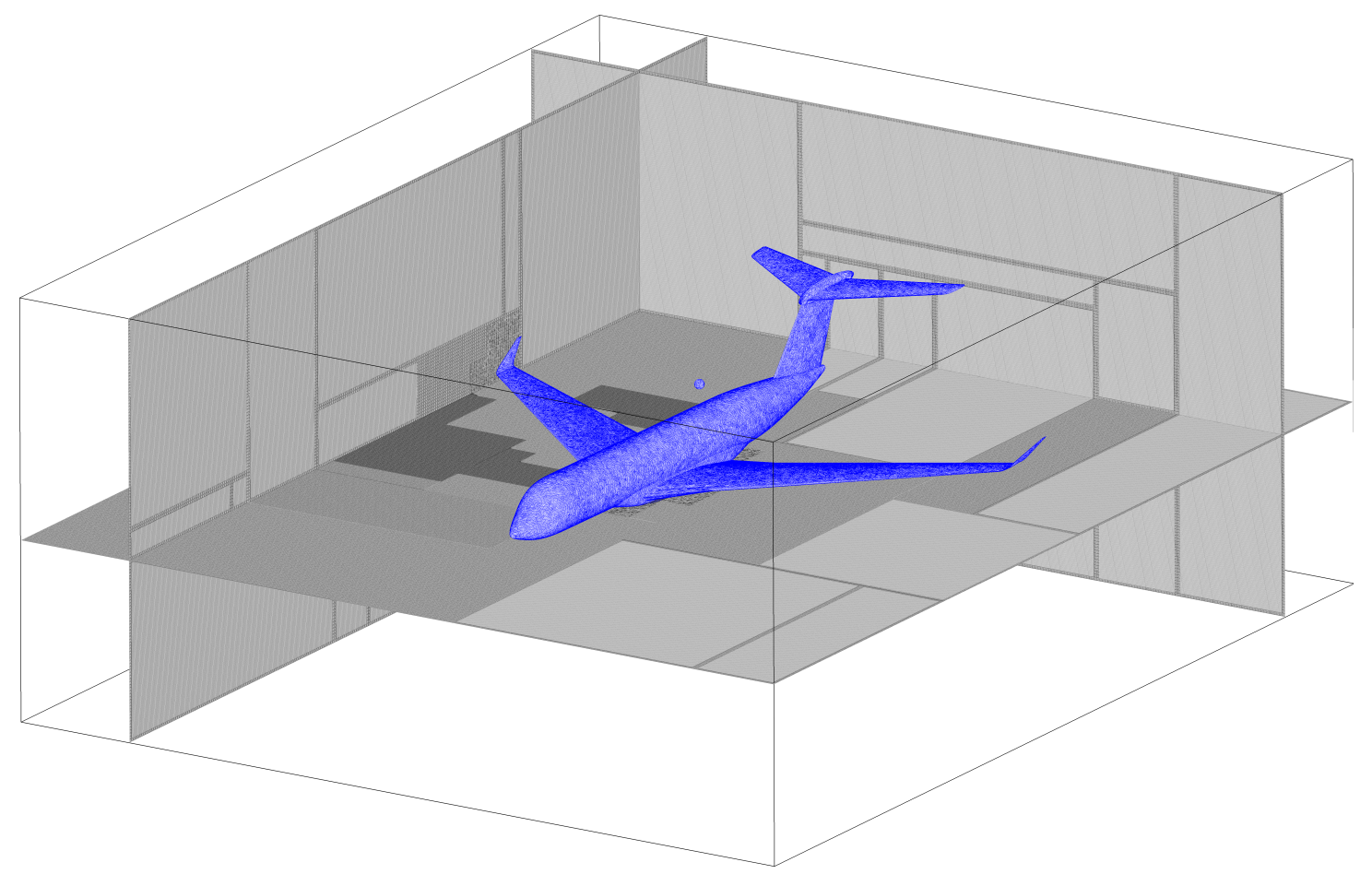

Fig. 14: Cut through hybrid DGM mesh used for aircraft scattering case

negative $\mathrm{x}$-direction. Table 5 shows the parameters of the DGM calculation. The computational domain was filled with 96 boxes leaving a tight fitting cavity for the geometry, cf. Fig. 15. The space be- tween the blocks and the geometry was filled with an unstructured mesh consisting of 911000 tetrahedra. The blocks were coupled by 9450000 surface tetrahedra. An equivalent purely cartesian mesh 
Table 5: Parameters of DGM aircraft calculation (8 Nodes)

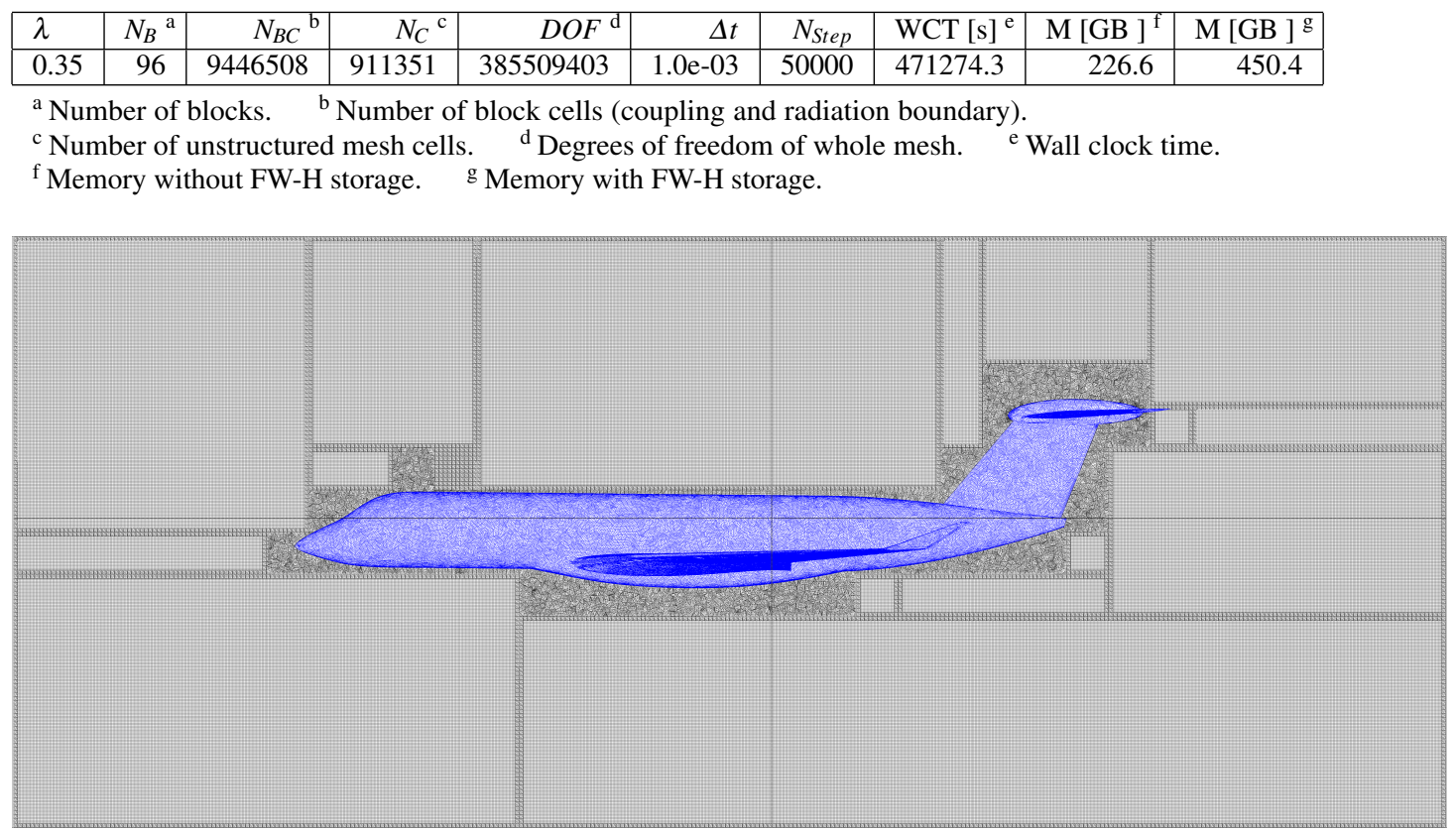

Fig. 15: Hybrid DGM mesh with tight fitting cavity around aircraft geometry

would have about $385 \times 10^{6}$ grid points. The mesh size of the cartesian blocks were 0.05 resolving a wavelength of $0.3 \mathrm{~m}$ with 7 points. The time step size, determined by the CFL limit of the smallest cells, would have been $1.42 \times 10^{-4}$ leading to quite lengthy calculations.

Fortunately, examination of the mesh revealed, that by dropping some small cells the time step could be increased to $1 \times 10^{-3}$ without any significant loss of accuracy. Neglection of small cells is easily implemented in the code by writing zeros into the cell variables at start of each RungeKutta sub step. Thus, the faces of neglected cells are treated as outflow boundaries where a small amount of acoustic energy is radiated out of the computational domain.

50000 time steps were performed to create a sufficiently long signal on the FW-H surface for extrapolation of the pressure field into the far-field. The FW-H surface is an ellipsoid with the origin $(15,0,0)$ and the half axes $(23,18,8)$, cf. Fig. 17a and Fig. 17b. It consists of 439000 triangles. The wall clock time of the calculation was about $132 \mathrm{~h}$ on 8 nodes (192 cores). The memory requirement for the hybrid solution alone was about $227 \mathrm{~GB}$ and included the FW-H time history data 450 GB.

\subsection{The FMM Calculation}

The parameters of the FMM calculation can be found in Table 6. For the FMM calculation the surface was discretized with 485000 triangles, yielding a resolution of 10.7 elements per wavelength. The FMM calculation needed 13 GMRES steps for convergence and lastet about 430 [s] on 16 cores. This is about 3 orders of magnitude faster than the DGM. Fig. 16 depicts the the real part of the surface pressure in case of the FMM Fig. 16a and a snapshot of the pressure in the DGM case Fig. 16b. The surface pressure pattern is very similar in both cases. Fig. 17c depicts the RMS value of the pressure $120 \mathrm{~m}$ below the aircraft centerline together with the data of the monopole source alone. The FMM and DGM results are in very good agree- 
Table 6: Parameters of FMM aircraft calculation

\begin{tabular}{|l|c|c|c|c|c|}
\hline$\lambda$ & $N_{E}{ }^{\mathrm{a}}$ & $p p w^{\mathrm{b}}$ & $N_{\text {iter }}{ }^{\mathrm{c}}$ & $\left.\mathrm{WCT}^{\mathrm{W}}\right]^{\mathrm{d}}$ & $\mathrm{M}[\mathrm{MB}]^{\mathrm{e}}$ \\
\hline 0.35 & 485308 & 10.68 & 13 & 428.10 & 5618.00 \\
\hline
\end{tabular}

a Number of elements. ${ }^{\mathrm{b}}$ Elements per wavelength.

${ }^{\mathrm{c}}$ Number of GMRES iterations. ${ }^{\mathrm{d}}$ Wall clock time on 1

node. e Memory.

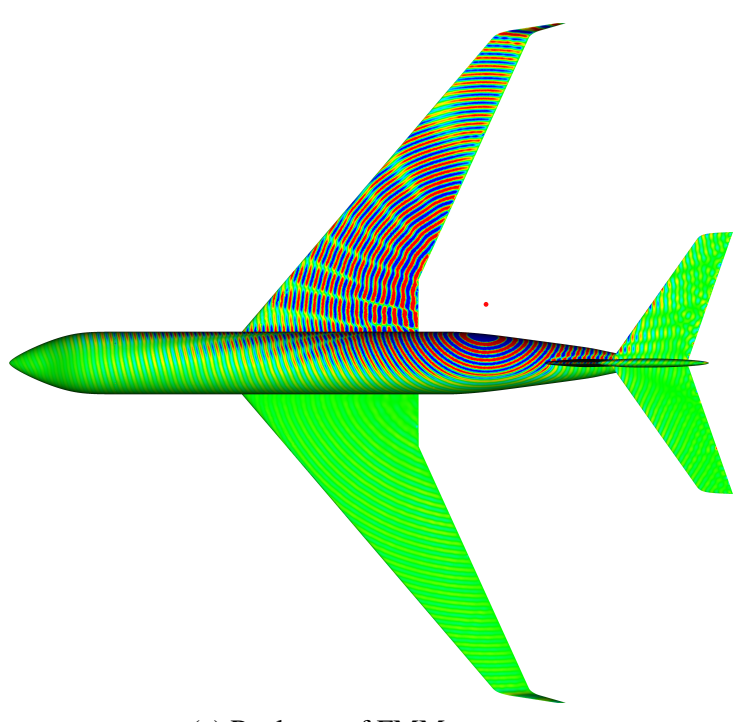

(a) Real part of FMM pressure

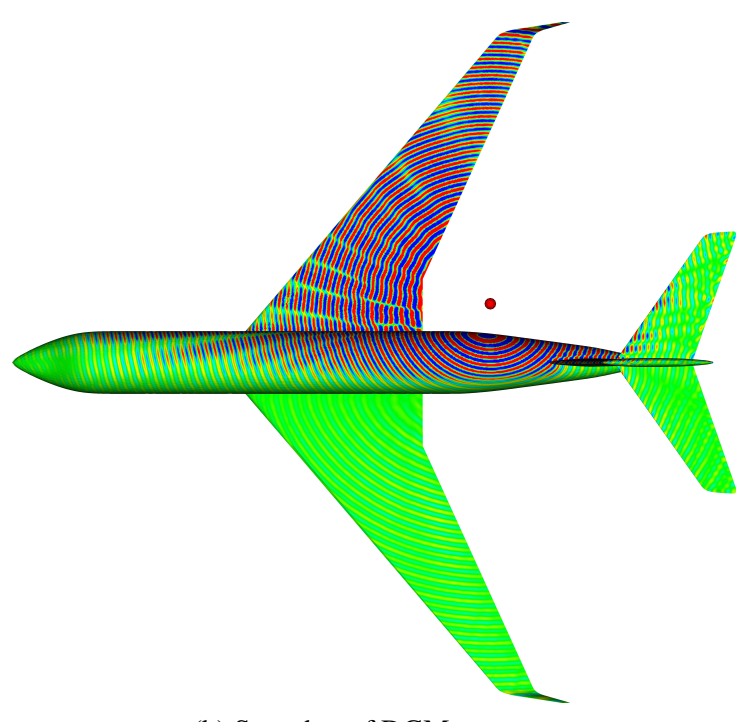

(b) Snapshot of DGM pressure

Fig. 16: Surface pressures (Monopole position marked by red spheres)

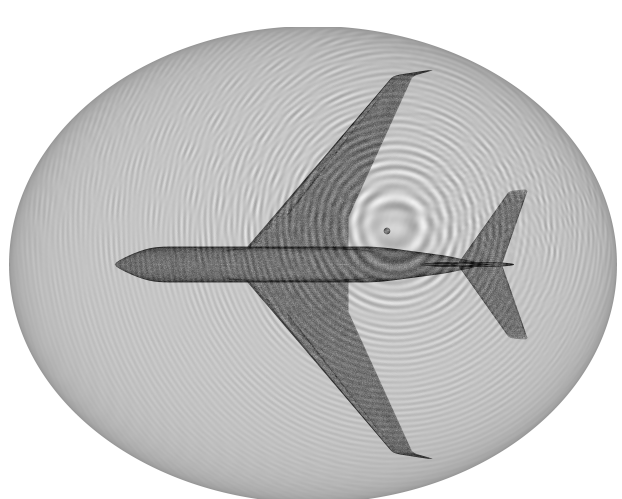

(a) $(x, y)$ plane

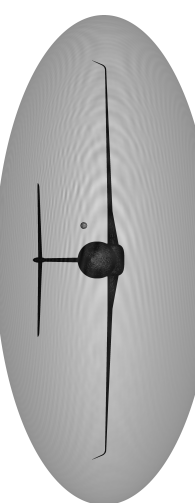

(b) $(y, z)$ plane

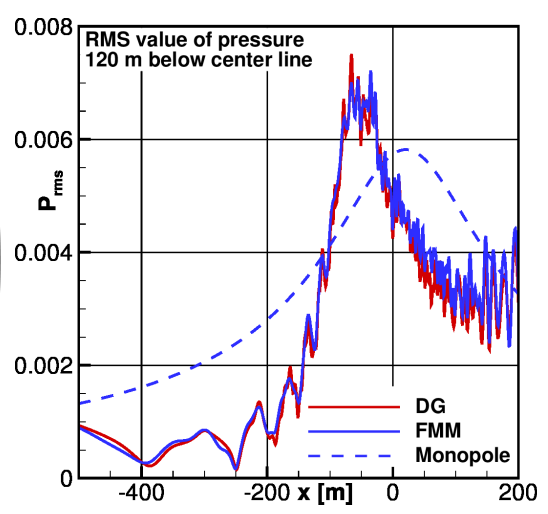

(c) RMS value of pressure

Fig. 17: FW-H surface and RMS value of pressure $120 \mathrm{~m}$ below aircraft centerline

ment. In the range $-500 \lesssim x \lesssim 100 \mathrm{~m}$ the acoustic shielding by the wing is visible. 


\section{Conclusions}

Acoustic installation effects have been considered as scattering problem. Two methods to investigate them numerically have been presented. First, a fast multipole boundary element solution of the wave equation where low Mach number mean flow effects can be taken into account by a Taylor transformation and, second, a hybrid Discontinuous Galerkin Method which solves acoustic perturbation equations. The memory requirements of the DGM could be reduced significantly by a hybrid mesh approach.

While calculations of full scale aircraft geometries at realistic frequencies are yet beyond the practical limits of the DGM, it is already a valuable tool to assess the approximations made by simpler methods like, e. g., the FMM solution of the Taylortransformed wave equation. The DGM results were transferred into the far-field using a FW-H surface.

Presented test cases were the scattering of an acoustic monopole at a sphere and a flat plate. It has been demonstrated by comparison of FMM and DGM results that for Mach numbers up to about 0.2 the Taylor-transformed FMM gives sufficiently accurate solutions for scattering problems.

Then, the scattering of an $1 \mathrm{kHz}$ monopole at a full scale aircraft geometry of moderate size has been presented. Mean flow effects were not taken into account. The correspondence between FMM and DGM solution is quite impressive. However, although the DGM time step size could be increased by a factor of 7 by dropping some small cells, the wall clock time of the calculation was about 130 h. A further speed up would require, e. g., the use of Nonuniform-Time-Stepping (NUTS) [11,31], or some implicit RK-methods, to get rid of the CFL time step limit.

Finally, it should be emphasized, that the FMM and DGM codes are by no means restricted to monopole scattering. Using appropriate source surfaces also realistic sound fields, like, e. g., fan noise, can be fed into the codes and its scattering at airframes be calculated. So far, the FMM seems to be fast and accurate enough to be used for the assessment of engine noise installation effects already in the design phase of aircrafts.
It should be mentioned, however, that while the FMM runs significantly faster than the DGM, the assumption of a low Mach number potential mean flow is often not fulfilled for complex flows. In this case one has to fall back on the far more expensive DGM which can deal with arbitrary mean flow fields.

\section{References}

1. Agarwal, A., Dowling, A.P.: Low-frequency acoustic shielding by the silent aircraft airframe. AIAA Journal 45(2), 358-365 (2007)

2. Amini, S.: On the choice of the coupling parameter in boundary integral formulations of the exterior acoustic problem . Applicable analysis 35(1), 75-92 (1990)

3. Astley, R.J., Bain, J.G.: A three-dimensional boundary element scheme for acoustic radiation in low mach number flows. Journal of Sound and Vibration 109(3), 445-465 (1986)

4. Atkins, H.L., Shu, C.W.: Quadrature-free implementation of discontinuous Galerkin method for hyperbolic equations. AIAA journal 36(5), 775-782 (1998)

5. Balay, S., Abhyankar, S., Adams, M.F., Brown, J., Brune, P., Buschelman, K., Dalcin, L., Eijkhout, V., Gropp, W.D., Kaushik, D., Knepley, M.G., McInnes, L.C., Rupp, K., Smith, B.F., Zampini, S., Zhang, H., Zhang, H.: PETSc users manual. Tech. Rep. ANL-95/11 - Revision 3.7, Argonne National Laboratory (2016). URL http: //www.mcs.anl.gov/petsc

6. Balay, S., Abhyankar, S., Adams, M.F., Brown, J., Brune, P., Buschelman, K., Dalcin, L., Eijkhout, V., Gropp, W.D., Kaushik, D., Knepley, M.G., McInnes, L.C., Rupp, K., Smith, B.F., Zampini, S., Zhang, H., Zhang, H.: PETSc Web page (2016). URL http: //www.mcs.anl.gov/petsc

7. Balay, S., Gropp, W.D., McInnes, L.C., Smith, B.F.: Efficient management of parallelism in object oriented numerical software libraries. In: E. Arge, A.M. Bruaset, H.P. Langtangen (eds.) Modern Software Tools in Scientific Computing, pp. 163-202. Birkhäuser Press (1997)

8. Balin, N., Casenave, F., Dubois, F., Duceau, E., Duprey, S., Terrasse, I.: Boundary element and finite element coupling for aeroacoustics simulations. Journal of Computational Physics 294, 274-296 (2015). DOI 10.1016/j. jcp.2015.03.044. https://doi.org/10.1016/j.jcp. 2015.03 .044

9. Balin, N., Sylvand, G., Robert, J.: Fast methods applied to BEM solvers for acoustic propagation problems. In: 22nd AIAA/CEAS Aeroacoustics Conference. American Institute of Aeronautics and Astronautics (2016). DOI 10.2514/6.2016-2712. https: //doi.org/10.2514/6.2016-2712

10. Bauer, M.: Airframe noise prediction using a discontinuous Galerkin method. Forschungsbericht 2011-11, 
Deutsches Zentrum für Luft- und Raumfahrt, Institut für Aerodynamik und Strömungstechnik (2011)

11. Bauer, M., Dierke, J., Ewert, R.: On the performance of airframe noise prediction on unstructured grids. Tech. Rep. AIAA-2012-2148, 18th AIAA/CEAS Aeroacoustics Conference, Colorado Springs, Colorado, USA (June 04-06, 2012)

12. Berntsen, J., Espelid, T.O.: Algorithm 706: DCUTRI An algorithm for adaptive cubature over a collection of triangles. ACM Transactions on Mathematical Software (TOMS) 18(3), 329-342 (1992)

13. Burton, A.J., Miller, G.F.: The application of integral equation methods to the numerical solution of some exterior boundary value problems. Proceedings of the Royal Society, London A 323, 201-210 (1971)

14. Carayol, Q., Collino, F.: Error estimates in the fast multipole method for scattering problems part 2: Truncation of the gegenbauer series. ESAIM: Mathematical Modelling and Numerical Analysis 39(1), 183-221 (2005)

15. Coifman, R., Rokhlin, V., Wandzura, S.: The fast multipole method for the wave equation: A pedestrian prescription. IEEE Antennas and Propagation Magazine 35(3), 7-12 (1993)

16. Delfs, J.: Grundlagen der Aeroakustik (Basics of Aeroacoustics) (2016). http://www.dlr.de/ as/en/desktopdefault.aspx/tabid-191/401_ read-22566

17. Delnevo, A., Le Saint, S., Sylvand, G., Terrasse, I.: Numerical methods: Fast multipole method for shielding effects. In: 11th AIAA/CEAS Aeroacoustics Conference. American Institute of Aeronautics and Astronautics (2005). DOI 10.2514/6.2005-2971. https: //doi.org/10.2514/6.2005-2971

18. Dunn, M., Tinetti, A.: Application of fast multipole methods to the NASA fast scattering code. In: 14th AIAA/CEAS Aeroacoustics Conference (29th AIAA Aeroacoustics Conference). American Institute of Aeronautics and Astronautics (2008). DOI 10.2514/6.2008-2875. https://doi.org/10.2514/6. 2008-2875

19. Ewert, R., Schröder, W.: Acoustic perturbation equations based on flow decomposition via source filtering. Journal of Computational Physics 188, 365-398 (2003)

20. Farassat, F.: Theory of noise generation from moving bodies with an application to helicopter rotors. Tech. Rep. NASA TR R-451 (1975)

21. Ffowcs Williams, J.E., Hawkings, D.L.: Sound generation by turbulence and surfaces in arbitrary motion. Philosophical Transactions of the Royal Society of London A: Mathematical, Physical and Engineering Sciences 264(1151), 321-342 (1969)

22. Francescantonio, P.D.: A new boundary integral formulation for the prediction of sound radiation. Journal of Sound and Vibration 202(4), 491-509 (1997)

23. Geuzaine, C., Remacle, J.F.: Gmsh: A 3-d finite element mesh generator with built-in pre- and post-processing facilities. International Journal for Numerical Methods in Engineering 79(11), 1309-1331 (2009)
24. Hu, F.Q.: An efficient solution of time domain boundary integral equations for acoustic scattering and its acceleration by graphics processing units. In: 19th AIAA/CEAS Aeroacoustics Conference. American Institute of Aeronautics and Astronautics (2013). DOI 10.2514/6.2013-2018. https://doi.org/10.2514/6. 2013-2018

25. Hu, F.Q., Pizzo, M.E.: On the assessment of acoustic scattering and shielding by time domain boundary integral equation solutions. In: 22nd AIAA/CEAS Aeroacoustics Conference. American Institute of Aeronautics and Astronautics (2016). DOI 10.2514/6.2016-2779. https://doi.org/10.2514/6.2016-2779

26. Hu, F.Q., Pizzo, M.E., Nark, D.M.: A new formulation of time domain boundary integral equation for acoustic wave scattering in the presence of a uniform mean flow. In: 23rd AIAA/CEAS Aeroacoustics Conference. American Institute of Aeronautics and Astronautics (2017). DOI 10.2514/6.2017-3510. https: //doi.org/10.2514/6.2017-3510

27. Karypis, G., Kumar, V.: A fast and high quality multilevel scheme for partitioning irregular graphs. SIAM Journal on Scientific Computing 20(1), 359-392 (1999)

28. Kirchhoff, G.: Zur Theorie der Lichtstrahlen. Annalen der Physik 254(4), 663-695 (1883)

29. Kleinman, R., Kress, R.: On the condition number of integral equations in acoustics using modified fundamental solutions. IMA Journal of applied mathematics 31(1), 79 (1983)

30. Laursen, M.E., Gellert, M.: Some criteria for numerically integrated matrices and quadrature formulas for triangles. International Journal for Numerical Methods in Engineering 12, 67-76 (1978)

31. Liu, L., Li, X., Hu, F.Q.: Nonuniform time-step rungekutta discontinuous Galerkin method for computational aeroacoustics. Journal of Computational Physics 229(19), 6874-6897 (2010)

32. Lummer, M.: A hybrid 3d discontinuous Galerkin code for CAA applications. In: 22nd AIAA/CEAS Aeroacoustics Conference, 30 May - 1 June, 2016, Lyon, France. DOI 10.2514/6.2016-2719. http://dx.doi. org/10.2514/6.2016-2719

33. Lummer, M., Richter, C., Pröber, C., Delfs, J.: Validation of a model for open rotor noise predictions and calculation of shielding effects using a fast bem. In: 19th AIAA/CEAS Aeroacoustics Conference, May 27-29, 2013, Berlin, Germany. DOI 10.2514/6.2013-2096. http://arc.aiaa.org/doi/ abs/10.2514/6.2013-2096

34. Mayoral, S., Papamoschou, D.: Prediction of jet noise shielding with forward flight effects. Tech. Rep. AIAA2013-0326, 51th AIAA Aeroaspace Sciences Meeting including the New Horizons Forum and Aerospace Exposition, Grapevine, Texas, USA (January 7-10, 2013)

35. Najafi-Yazdi, A., Brès, G.A., Mongeau, L.: An acoustic analogy formulation for moving sources in uniformly moving media. Proceedings of the Royal Society of London A: Mathematical, Physical and Engineering Sciences 467(2125), 144-165 (2011) 
36. Nark, D.M., Jones, M.G.: Development of a multifidelity approach to acoustic liner impedance eduction. In: 23rd AIAA/CEAS Aeroacoustics Conference. American Institute of Aeronautics and Astronautics (2017). DOI 10.2514/6.2017-3181. https: //doi.org/10.2514/6.2017-3181

37. Orszag, S.A.: Fourier series on spheres. Monthly Weather Review 102, 56-75 (1974)

38. Rahola, J.: Diagonal forms of the translation operators in the fast multipole algorithm for scattering problems. BIT Numerical Mathematics 36(2), 333-358 (1996)

39. Richter, C., Hay, J.A., łukasz Panek, Schönwald, N., Busse, S., Thiele, F.: A review of time-domain impedance modelling and applications. Journal of Sound and Vibration 330(16), 3859-3873 (2011). DOI 10.1016/j.jsv.2011.04.013. https://doi.org/ 10.1016/j.jsv.2011.04.013

40. Richter, C., Thiele, F.H., Li, X.D., Zhuang, M.: Comparison of time-domain impedance boundary conditions for lined duct flows. AIAA Journal 45(6), 1333-1345 (2007). DOI 10.2514/1.24945. https://doi.org/10. 2514/1.24945

41. Shenderov, E.L.: Diffraction of a sound wave by the open end of a flanged waveguide with impedance walls. Acoustical Physics 46(6), 716-727 (2000)

42. $\mathrm{Si}, \mathrm{H}$.: Tetgen, a delaunay-based quality tetrahedral mesh generator. ACM Transactions on Mathematical Software (TOMS) 41(2), Article 11, 1-36 (2015)

43. Steger, J.L., Warming, R.F.: Flux vector splitting of the inviscid gasdynamic equations with application to finite-difference methods. Journal of Computational Physics 40, 263-293 (1981)

44. Sylvand, G.: Performance of a parallel implementation of the FMM for electromagnetics applications. International Journal for Numerical Methods in Fluids 43(8), 865-879 (2003)

45. Tam, C.K.W., Auriault, L.: Time-domain impedance boundary conditions for computational aeroacoustics. AIAA Journal 34(5), 917-923 (1996). DOI 10.2514/3. 13168. https://doi.org/10.2514/3.13168

46. Tam, C.K.W., Webb, J.C.: Dispersion-relationpreserving finite difference schemes for computational acoustics. Journal of Computational Physics 107, 262-281 (1993)

47. Taylor, K.: A transformation of the acoustic equation with implications for wind-tunnel and low-speed flight tests. Proceedings of the Royal Society London A 363, 271-281 (1978)

48. Tinetti, A., Dunn, M.: Aeroacoustic noise prediction using the fast scattering code. In: 11th AIAA/CEAS Aeroacoustics Conference. American Institute of Aeronautics and Astronautics (2005). DOI 10.2514/6.2005-3061. https://doi.org/10.2514/6. 2005-3061

49. Tinetti, A., Dunn, M.: Acoustic simulations of an installed tandem cylinder configuration. In: 15th AIAA/CEAS Aeroacoustics Conference (30th AIAA Aeroacoustics Conference). American Institute of Aeronautics and Astronautics (2009). DOI 10.
2514/6.2009-3158. https://doi.org/10.2514/6. 2009-3158

50. Tinetti, A., Dunn, M.: Scattering of high frequency duct noise by full scale hybrid wing body configurations. In: 15th AIAA/CEAS Aeroacoustics Conference (30th AIAA Aeroacoustics Conference). American Institute of Aeronautics and Astronautics (2009). DOI 10.2514/6.2009-3400. https://doi.org/10.2514/6. 2009-3400

51. Wolf, W.R., Lele, S.K.: Wideband fast multipole boundary element method: Application to acoustic scattering from aerodynamic bodies. International Journal for $\mathrm{Nu}$ merical Methods in Fluids 67(12), 2108-2129 (2011)

52. Wolf, W.R., Lele, S.K.: Fast acoustic scattering predictions with non-uniform potential flow effects. Journal of the Brazilian Society of Mechanical Sciences and Engineering 35(4), 407-418 (2013)

53. Wolf, W.R., Lele, S.K.: Fast acoustic scattering simulations with non-uniform potential flow effects. Tech. Rep. AIAA-2010-3712, 16th AIAA/CEAS Aeroacoustics Conference, Stockholm, Sweden (June 7-9, 2010)

54. Wolf, W.R., Lele, S.K.: Assessment of fast multipole method formulations for acoustic scattering. Tech. Rep. AIAA-2009-3229, 15th AIAA/CEAS Aeroacoustics Conference, Miami, Florida (May 11-13, 2009)

55. Zienkiewicz, O.C., Taylor, R.L.: The Finite Element Method, Fifth edition, Volume1: The Basis. Butterworth-Heinemann, Oxford (2000) 